\title{
TRANSFINITE DUALS OF QUASIREFLEXIVE BANACH SPACES
}

\author{
BY
}

\author{
STEVEN F. BELLENOT
}

\begin{abstract}
The transfinite duals of a space with a neighborly basis are constructed until they become nonseparable. Let $s(X)$ be the first ordinal $\alpha$ so that $X^{\alpha}$ is nonseparable. It is shown that if $X$ is nonreflexive, $s(X) \leqslant \omega^{2}+1$ (this is best possible) and that $\{s(X): X$ separable quasireflexive of order one $\}=\{\omega+1, \omega+$ $\left.2,2 \omega+1,2 \omega+2, \omega^{2}+1\right\}$. A quasireflexive space $X$ is constructed so that $X^{\omega}$ is isomorphic to $X \oplus c_{0}$ and no basic sequence in $X$ is equivalent to a neighborly basis. It is shown that the $\omega^{2}$ th dual of James space and James function space are isomorphic to subspaces of one another. Also, perhaps of interest on its own, a reflexive space with a subsymmetric basis is constructed whose inversion spans a nonreflexive space.
\end{abstract}

The notions of transfinite duals and quasireflexive spaces are more intimately related than one might expect. Indeed, Perrott in [14] has shown that for many "natural" sequences $\left\{x_{n}\right\}$ in the transfinite dual $X$ of a nonreflexive space $X$, the space $\left[x_{n}\right]$ has a structure which often makes it quasireflexive. The author in [2] turned this around and used results about quasireflexive spaces (actually of the $\left\{x_{n}\right\}$ ) to obtain results about $X^{\omega}$ and hence $X$. Thus the goal of this paper is to describe the transfinite duals of quasireflexive spaces and to see what these results imply about the transfinite duals of an arbitrary nonreflexive space.

Our vehicle for this goal is the notion of a neighborly basis, defined by James in [8]. (The basic properties of neighborly bases are in $§ 1$.) There are three reasons for restricting attention to neighborly bases. First, the result of Perrott mentioned above implies that if $X$ is quasireflexive of order one, then for some neighborly basic sequence $\left\{e_{n}\right\}, X^{\omega}$ is isomorphic to $X \oplus\left[e_{n}\right]$. The second is that (to the author's knowledge) all the concrete examples of quasireflexive spaces of order one in the literature can be renormed so to have a neighborly basis. (However, the examples in $\$ 4$ will make this statement no longer true.) The final justification is that we can isometrically describe all the transfinite duals of a space with a neighborly basis until they become nonseparable. (These results are in \$2.) Indeed, if both the neighborly basis $\left\{x_{n}\right\}$ and its inversion span quasireflexive spaces, we give three different isomorphic representations of the space $\left[x_{n}\right]^{\omega^{2}}$.

Define $s(X)$, for a separable nonreflexive space $X$, to be the first ordinal $\alpha$ so that $X^{\alpha}$ is nonseparable. As applications, it follows that $s(X) \leqslant \omega^{2}+1$ (which improves

\footnotetext{
Received by the editors November 19, 1980 and, in revised form, May 11, 1981. Presented at Mathematiches Forschungsinstitut, Oberwolfach on August 7, 1981.

1980 Mathematics Subject Classification. Primary 46B10, 46B15.

Key words and phrases. Neighborly, equal signs additive, invariant under spreading, quasireflexive, transfinite duals, separable and nonseparable, James space, inversion of a basis.
} 
$s(X) \leqslant \omega^{2}+2$, given in Davis and Lindenstrauss [5]) and this is best possible. Also $\{s(X): X$ quasireflexive of order one $\}=\left\{\omega+1, \omega+2,2 \omega+1,2 \omega+2, \omega^{2}+1\right\}$. These results are in $\S 2$ and the examples are in $\S 4$.

In $\S 3$, we show that if $J$ (resp. $J F$ ) is James space (resp. James function space) then the spaces $J^{\omega^{2}}$ and $J F$ are closely related. Although these spaces might not be isomorphic, they are isomorphic to subspaces of one another and $J^{\omega^{2}}$ is isomorphic to a complemented subspace of $J F$. This construction is more general and "works" for all neighborly bases with a single exception. (See $\$ 3$ for details.) The author has been told that Perrott in [13] constructs $J^{\omega^{2}}$ (in a way different from ours) and shows that it has properties similar to $J F$.

Perhaps the most interesting part of this paper is $\$ 4$, which is devoted to examples. Of interest outside the context of this paper is the construction of a reflexive space with a subsymmetric basis whose inversion spans a nonreflexive space. There is a general construction (Theorem 4.3) which produces a concrete quasireflexive space $X$ of order one and a basis, so that $X^{\omega}$ is isomorphic to $X \oplus\left[e_{n}\right]$, where $\left\{e_{n}\right\}$ is any predetermined neighborly sequence with modest restrictions. These two results combine to produce the quasireflexive spaces $X$ with $s(X)=\omega+1, \omega+2,2 \omega+1$ and $2 \omega+2$. Another application of Theorem 4.3 produces a quasireflexive space $X$ so the $X^{\omega}$ is isomorphic to $X \oplus c_{0}$. This space has the property that no basic sequence in $X$ (nor any basis of a quotient of $X$ ) is equivalent to a neighborly sequence.

0. Preliminaries. All our spaces are normed vector spaces over either the real or complex field. The main reference is [12], where undefined terms may be found. In particular, see [12] for the definitions and properties of monotone, shrinking, boundedly complete, equivalent, unconditional, subsymmetric and symmetric bases and basic sequences. We do not require that a basis or basic sequence be normalized.

Our notation also generally agrees with [12]. In particular, we write $\left\{x_{n}\right\}$ or $\left\{x_{n}\right\}_{n}$ for $\left\{x_{n}\right\}_{n=1}^{\infty}, \sum x_{n}$ or $\sum_{n} x_{n}$ for $\sum_{n=1}^{\infty} x_{n}$, and $\left[x_{n}\right]$ (resp. $\left.\left[x_{n}\right]_{n=1}^{m}\right)$ for the closed linear span of $\left\{x_{n}\right\}$ (resp. $\left\{x_{n}\right\}_{n=1}^{m}$ ).

A basic sequence $\left\{e_{n}\right\}$ is said to be orthogonal (resp. bimonotone) if, for each finitely nonzero scalar sequence $\left\{\alpha_{n}\right\}$ and for each subset of the positive integers $F$ (resp. $F=\{m, m+1, \ldots, m+p\}, m, p \geqslant 0)$,

$$
\left\|\sum \alpha_{n} e_{n}\right\| \geqslant\left\|\sum_{n \in F} \alpha_{n} e_{n}\right\| .
$$

An orthogonal sequence is unconditional with unconditional constant $\leqslant 4$. A sequence $\left\{e_{n}\right\}$ is invariant under spreading if for each finitely nonzero scalar sequence $\left\{\alpha_{n}\right\}$ and increasing sequence of positive integers $\{i(n)\}$

$$
\left\|\sum \alpha_{n} e_{n}\right\|=\left\|\sum \alpha_{n} e_{i(n)}\right\|
$$

A block basic sequence of $\left\{e_{n}\right\}$ (resp. skipped block basic sequence) is any sequence of nonzero vectors $\left\{b_{i}\right\}$ that can be written

$$
b_{i}=\sum_{n=n(i-1)+1}^{n(i)} \alpha_{n} e_{n}
$$


for some scalar sequence $\left\{\alpha_{n}\right\}$ and increasing sequence of positive integers $\{n(i)\}_{i=0}^{\infty}$ (resp. with the additional property that $\alpha_{n(i)}=0$, for each $i$ ).

A space $X$ is quasireflexive (of order $n$ ) if the canonical image of $X$ in its bidual is of finite codimension (resp. $n$-codimensional).

If the basic sequence $\left\{e_{n}\right\}$ has the property that $\left\|\sum \alpha_{n} e_{n}\right\|=\left\|\sum \alpha_{n} e_{n+k}\right\|$ for each positive integer $k$ (translation invariance), then we define the norm on $\left\{e_{n}\right\}_{n=-\infty}^{0}$ by

$$
\left\|\sum_{n=-m}^{0} \alpha_{n} e_{n}\right\|=\left\|\sum_{n=-m}^{0} \alpha_{n} e_{n+m+1}\right\| .
$$

The space $\left\{e_{n}\right\}_{n=-\infty}^{0}$ with this norm is called the inversion of $\left\{e_{n}\right\}$. If $\left\{e_{-n}\right\}_{n=1}^{\infty}$ is equivalent to $\left\{e_{n}\right\}_{n=1}^{\infty}$, we will say that $\left\{e_{n}\right\}$ is inversion invariant.

We will write ordinals $\left(\leqslant \omega^{2}+1\right)$ so that $n \omega$ is the limit of $(n-1) \omega+m$ as $m=1,2, \ldots$ for each positive integer $m$. The ordinal $\alpha+1$ is the successor to $\alpha$ and $\omega^{2}$ is the limit of $n \omega$ as $n$ runs through the positive integers. An ordinal $\alpha$ is said to be even if $\alpha=\beta+m$ where $\beta$ is either zero or a limit ordinal and $m$ is an even nonnegative integer.

The transfinite duals of a Banach space $X$ are defined for each ordinal by induction as follows. First, $X^{0}=X$ and $X^{\alpha+1}=\left(X^{\alpha}\right)^{\prime}$, the continuous dual of $X^{\alpha}$. If $\beta$ is a limit ordinal, $X^{\beta}$ is defined to be the completion of $\cup\left\{X^{\alpha}: \alpha<\beta\right.$, $\alpha$ even $\}$. (We indentify $X^{\alpha}$ with its canonical image in $X^{\alpha+2}$.) If $T: X \rightarrow Y$ is a continuous linear map, then for each even ordinal $\alpha$, there is a canonical map $T^{(\alpha)}: X^{\alpha} \rightarrow Y^{\alpha}$ also defined by induction. The map $T^{(\alpha+2)}$ is the double transpose of $T^{(\alpha)}$, and if $\beta$ is a limit ordinal $T^{(\beta)}$ is the unique extension of $T^{(\alpha)}: X^{\alpha} \rightarrow Y^{\alpha}$ for each even $\alpha<\beta$. In particular, if $J: X \rightarrow X^{2}$ is the canonical injection, let $S=J^{(\omega)}$. Perrott's result [14] mentioned above is if $x_{1} \in X^{2} / X$ and $x_{n+1}=S x_{n}$, then $\left\{x_{n}\right\}$ is ESA in the sense of this paper (see §2).

Finally we state Proposition 1.b.2 of [12] because we will use it often. If $\left\{e_{n}\right\}$ is a monotone shrinking basis, then $\left[e_{n}\right]^{2}$ is isometric with the set of all sequences $\left\{\alpha_{n}\right\}$ with norm $\left\|\left\{\alpha_{n}\right\}\right\|=\sup _{n}\left\|\sum_{i=1}^{n} \alpha_{i} e_{i}\right\|<\infty$. Furthermore the map $\sum \alpha_{n} e_{n} \rightarrow\left\{\alpha_{n}\right\}$ from $\left[e_{n}\right]$ to $\left[e_{n}\right]^{2}$ is the canonical injection.

1. Neighborly bases and norms. The reader is warned that the definition of neighborly basis is slightly different than that in [8]. However, they are the same if we allow the space to be renormed.

Definition. A sequence $\left\{e_{n}\right\}$ in a normed space $(X,\|\cdot\|)$ is said to be neighborly if for each finite nonzero sequence of scalars $\left\{\alpha_{n}\right\}$ and positive integer $k$, the norm of $\sum \alpha_{n} e_{n}$ is not increased by replacing $\alpha_{k}$ with either $\alpha_{k-1}$ or $\alpha_{k+1}$. (The sequence begins with $e_{1}$ and we use the convention that $\alpha_{0}$ is always zero.) And we will call $\|\cdot\|$ a neighborly norm.

It is known [8] and easy to check that a neighborly sequence $\left\{e_{n}\right\}$ with dense linear span is a bimonotone basis which satisfies each of the following inequalities for each finitely nonzero sequence of scalars $\left\{\alpha_{n}\right\}$, each increasing sequence of positive integers $\{i(n)\}$ and each positive integer $k$ :

(i) $\left\|\sum \alpha_{n} e_{n}\right\|=\left\|\sum_{1}^{k} \alpha_{n} e_{n}+\alpha_{k} e_{k+1}+\sum_{k+1}^{\infty} \alpha_{n} e_{n+1}\right\|$,

(ii) $\left\|\sum \alpha_{n} e_{n}\right\|=\left\|\sum \alpha_{n} e_{n+k}\right\|$,

(iii) $\left\|\sum \alpha_{n} e_{n}\right\| \geqslant\left\|\sum \alpha_{i(n)} e_{n}\right\|$. 
These conditions have names. Condition (i) is called repetition invariance, (ii) is called translation invariance and (iii) is called decreasing under sections. It is easy to check that the conditions (i), (ii) and (iii) together imply neighborly.

Two reasons for studying neighborly bases and norms are (1) that there is a nice way of extending the norm to a space of functions on any linearly ordered set and (2) the Projection Principle.

Let $L$ be a linearly ordered set. If $I \subset L$ has the property that $i, j \in I, k \in L$ and $i<k<j$ implies $k \in I$, then $I$ will be called an interval of $L$. Intervals are disjoint if they have a void intersection. Let $\left(\left\{e_{n}\right\},\|\cdot\|\right)$ be a neighborly basis and norm. If $f$ is a scalar-valued function on $L$, define the norm of $f$ by

$$
\|f\|=\sup \left\{\left\|\sum_{i=1}^{n} f(j(i)) e_{i}\right\|: j(i) \in L, j(i)<j(i+1), n \geqslant 1\right\} .
$$

Let $F$ be the collection of functions on $L$ with finite norm.

It is straightforward to check that $\|\cdot\|$ is indeed a norm and that with this norm $F$ is complete. Furthermore, condition (iii) implies that if $L$ is the integers in their usual order, then the old norm and new norm agree on any finitely nonzero sequence. The space $F$ has lots of norm one projections. Indeed we have

The Projection Principle. If $f, g \in F$ and $\left\{I_{\alpha}\right\}_{\alpha \in \Lambda}$ and $\left\{J_{\alpha}\right\}_{\alpha \in \Lambda}$ are pairwise disjoint collections of intervals of $L$ so that for each $\alpha \in \Lambda, I_{\alpha} \supsetneq J_{\alpha}$ and $g$ restricted to $I_{\alpha}$ is constant and the support of $f$ is contained in $\cup_{\alpha \in \Lambda} J_{\alpha}$, then $\|f+g\| \geqslant\|g\|$.

For $\alpha \in \Lambda$, pick $i_{\alpha} \in I_{\alpha} \backslash J_{\alpha}$ and define $\phi: L \rightarrow L$ by $\phi(j)=i_{\alpha}$ if $j \in I_{\alpha}$ and otherwise $\phi(i)=i$. Let $j(i) \in L, j(i)<j(i+1)$ for $1 \leqslant i \leqslant n$. We have $\phi$ is orderpreserving and

$$
\left\|\sum_{1}^{n} g(j(i)) e_{i}\right\|=\left\|\sum_{1}^{n} g(\phi(j(i))) e_{i}\right\|=\left\|\sum_{1}^{n}(f+g)(\phi(j(i))) e_{i}\right\| .
$$

Taking sups yields $\|f+g\| \geqslant\|g\|$.

The next lemma is an aid in actually computing the norms of functions in $F$. It shows that neighborly norms have some "variational" properties. It also shows that if $f$ is a continuous piecewiese linear function on $L=[0,1]$, we can compute the norm of $f$ by restricting the $j(i)$ 's to the "corners" of $f$ (i.e. points $i$ where $f^{\prime}(i)$ does not exist).

LEMMA 1.1. Let $\left\{e_{n}\right\}$ be neighborly and let $\left\{\alpha_{n}: n \geqslant 1\right\}$ be a finitely nonzero sequence. Then (with the convention that $\alpha_{0}=0$ ),

(a) if for some $k \geqslant 1, \alpha_{k}$ is a convex combination of $\alpha_{k-1}$ and $\alpha_{k+1}$, then

$$
\left\|\sum \alpha_{n} e_{n}\right\|=\left\|\sum_{1}^{k-1} \alpha_{n} e_{n}+\sum_{k+1}^{\infty} \alpha_{n} e_{n-1}\right\|
$$

(b) if $k \geqslant 1$ and $b$ is a convex combination of $\alpha_{k-1}$ and $\alpha_{k}$, or a convex combination of $\alpha_{k}$ and $\alpha_{k+1}$, then

$$
\left\|\sum \alpha_{n} e_{n}\right\| \geqslant\left\|\sum_{1}^{k-1} \alpha_{n} e_{n}+b e_{k}+\sum_{k+1}^{\infty} \alpha_{n} e_{n}\right\|
$$


(c) if for some $k \geqslant 1$ and scalars $b$ and $c$, the numbers $b, \alpha_{k}, \alpha_{k+1}$ and c are collinear in that order, then

$$
\left\|\sum \alpha_{n} e_{n}\right\| \leqslant\left\|\sum_{1}^{k-1} \alpha_{n} e_{n}+b e_{k}+c e_{k+1}+\sum_{k=2}^{\infty} \alpha_{n} e_{n}\right\| .
$$

Proof. (a) Decreasing under sections shows one inequality. The other inequality follows since the left-hand element is a convex combination of the elements formed from the right-hand element by repeating either $\alpha_{k-1}$ or $\alpha_{k+1}$ and by use of repetition invariance.

The proof of (b) is another convex combination argument, this time using the neighborly property. And (c) follows from two applications of (b).

We collect some known properties about neighborly bases in the next proposition. Some proofs are given, both for completeness and later reference, otherwise only an outline is given.

Proposition 1.2. Let $\left\{e_{n}\right\}$ be a neighborly basis. Then:

(a) Each skipped block basic sequence is orthogonal.

(b) The sequence $\left\{e_{2 n}\right\}_{n}$ is invariant under spreading and hence is a subsymmetric basic sequence.

(c) Either $\left\{e_{n}\right\}$ is shrinking, or $\left\{e_{n}\right\}$ has a skipped block basic sequence equivalent to the usual basis of $l_{1}$.

(d) The formal sum $\sigma=\sum_{n=1}^{\infty} e_{n} \in\left[e_{n}\right]^{2}$, so that $\left[e_{n}\right]$ is never reflexive. If $\left\{e_{n}\right\}$ is shrinking, either $\left[e_{n}\right]^{2}=\left[\left\{e_{n}\right\}_{n=1}^{\infty} \cup\{\sigma\}\right]$ or $\left\{e_{n}\right\}$ has a skipped block basic sequence equivalent to the usual basis of $c_{0}$.

(e) If $f_{n}=\sum_{i=n}^{\infty} e_{i}$, then $\left[e_{n}\right]$ is isomorphic to $\left[f_{n}\right]$.

(f) If $\left[e_{n}\right]$ is quasireflexive, then $\left[e_{n}\right]$ is isomorphic to both $\left[e_{n}\right]^{2}$ and $\left[e_{n}\right] \oplus Y$, for each finite dimensional $Y$.

Proof. (a) Use neighborly to move the zero through a block.

(b) Use repetition invariance to repeat the zeros.

(c) If $\left\{e_{n}\right\}$ is not shrinking, then there is a continuous linear functional $F$, an $\varepsilon>0$, and a block basic sequence $\left\{b_{i}\right\}$ with $\left\|b_{i}\right\|=1$ and $F\left(b_{i}\right)>\varepsilon$. Then the skipped block basic sequence $\left\{b_{2 i}\right\}_{i}$ is equivalent to the usual basis of $l_{1}$ by (a).

(d) First, repetition invariance implies $\left\|\sum_{1}^{n} e_{i}\right\|=\left\|e_{1}\right\|<\infty$, hence $\sigma \in\left[e_{n}\right]^{2}$. If $\sum_{n=1}^{\infty} \alpha_{n} e_{n} \in\left[e_{n}\right]^{2} \backslash\left[\left\{e_{n}\right\} \cup\{\sigma\}\right]$, then by subtracting a suitable multiple of $\sigma$ we may assume the existence of a subsequence $\{n(i)\}$ so that $\lim _{i} \alpha_{n(i)}=0$. Thus by passing to a further subsequence and perhaps multiplying by a scalar, we may assume

$$
b_{i}=\sum_{n=n(i-1)+1}^{n(i)-1} \alpha_{n} e_{n}, \quad i \geqslant 2,
$$

satisfies $1<\left\|b_{i}\right\|$ and $\left\|\sum_{i=2}^{m} b_{i}+\sum_{i=2}^{m} \alpha_{n(i)} e_{n(i)}\right\|<2$, for all $m$, and that $\sum_{i=1}^{\infty}\left\|\alpha_{n(i)} e_{n(i)}\right\|<1 / 2$. From [12, p. 5], $\left\{b_{i}\right\}_{i=2}^{\infty}$ is equivalent to $\left\{b_{i}+\alpha_{n(i)} e_{n(i)}\right\}_{i=2}^{\infty}$ which is equivalent to the usual basis of $c_{0}$ by (a).

(e) If $x \in\left[f_{n}\right]$, then $x=\sum_{n=1}^{\infty} \alpha_{n} e_{n}$ and $\alpha_{\infty}=\lim \alpha_{n}$ exists. The map $S:\left[f_{n}\right] \rightarrow\left[e_{n}\right]$, defined by $S x=-\alpha_{\infty} e_{1}+\sum_{i=1}^{\infty}\left(\alpha_{i}-\alpha_{\infty}\right) e_{i+1}$, is the isomorphism whose inverse is $T:\left[e_{n}\right] \rightarrow\left[f_{n}\right]$, defined by $T\left(\sum \beta_{i} e_{i}\right)=\sum_{i=1}^{\infty}\left(\beta_{i+1}-\beta_{1}\right) e_{i}$. 
(f) The first statement combines (d) and (e) and the second follows by induction.

EXAMPLE 1.3. The following sequences are neighborly sequences with the given norm.

(a) The usual basis for $c_{0}$ with the sup norm.

(b) If $\left\{f_{n}\right\}$ is the usual basis for $l_{1}$, then $\left\{f_{n}-f_{n+1}\right\}_{n}$ is a neighborly sequence in the usual $l_{1}$-norm.

(c) The James space $J$. To make the usual unit vectors a neighborly basis we use the norm

$$
\begin{aligned}
& \left\|\left\{\boldsymbol{\alpha}_{n}\right\}\right\|=\sup \left\{\sum_{i=1}^{k}\left|\boldsymbol{\alpha}_{p(i)}-\alpha_{p(i+1)}\right|^{2}\right)^{1 / 2} \\
& \qquad \begin{array}{l}
0 \leqslant p(1)<p(2)<\cdots<p(k+1), k \geqslant 1\}
\end{array}
\end{aligned}
$$

(again we use the convention that $\alpha_{0}=0$ ).

(d) In [8], James constructed a quasireflexive space $X$ with a neighborly basis so that $X$ is isomorphic to $X^{\prime}$. He also showed that $X$ is not isomorphic to $J$.

(e) The uniformly nonoctahedral space in [7] and the related spaces $X_{\rho}, 1<\rho<\infty$, in [5 and 9] have the property that the usual vector basis is neighborly.

(f) The construction of $J$ was extended in [4 and 10] to the spaces $J(E)$, where $E$ is any Banach space with a symmetric basis $\left\{x_{n}\right\}$. The definition of the norm is given below in Proposition 1.4.

(g) All the above examples are inversion invariant. However Proposition 1.4 shows how to obtain $J(E)$ with $E$ having only a subsymmetric basis. Example 4.2 shows that the inversion of a neighborly basis can be quite different than the original basis.

Proposition 1.4. If $\|\cdot\|$ is a norm in which $\left\{e_{2 n}\right\}_{n}$ is orthogonal and invariant under spreading, and if for finitely nonzero scalar sequences $\left\{\alpha_{n}\right\}$ we define

$$
\rho\left(\sum_{n} \alpha_{n} e_{n}\right)=\sup \left\{\left\|\sum_{i=1}^{k}\left(\alpha_{p(i)}-\alpha_{p(i+1)}\right) e_{2 i}\right\|: 0 \leqslant p(1)<\cdots<p(k+1), k \geqslant 1\right\}
$$

with the convention $\alpha_{0}=0$, then $\left\{e_{n}\right\}$ is a neighborly sequence with respect to $\rho$ and $\rho$ restricted to $\left[e_{2 n}\right]_{n=1}^{\infty}$ is equivalent to $\|\cdot\|$.

Furthermore, if $\|\cdot\|$ is the restriction of a neighborly norm on $\left\{e_{n}\right\}$ to $\left[e_{2 n}\right]_{n}$, then $\rho\left(\sum \alpha_{n} e_{n}\right) \leqslant 2\left\|\sum \alpha_{n} e_{n}\right\|$.

ProOF. It is straightforward to check that $\rho$ is a norm. Showing $\left\{e_{n}\right\}$ is neighborly with respect to $\rho$ uses both facts that $\left\{e_{2 n}\right\}_{n}$ is orthogonal and invariant under spreading and is also straightforward. Since

$$
\begin{gathered}
\left\|\sum_{i=1}^{k}\left(\alpha_{p(i)}-\alpha_{p(i+1)}\right) e_{2 i}\right\| \leqslant\left\|\sum_{i=1}^{k} \alpha_{p(i)} e_{2 i}\right\|+\left\|\sum_{i=1}^{k} \alpha_{p(i+1)} e_{2 i}\right\|, \\
\rho\left(\sum_{n} \beta_{n} e_{2 n}\right) \leqslant 2 \\
\rho \sum_{n} \beta_{n} e_{2 n} \| . \text { Taking } p(i)=i-1, \\
\rho\left(\sum_{n} \beta_{n} e_{2 n}\right) \geqslant\left\|\sum_{n}\left(-\beta_{n} e_{4 n-2}+\beta_{n} e_{4 n}\right)\right\| \geqslant\left\|\sum_{n} \beta_{n} e_{2 n}\right\| .
\end{gathered}
$$


To see the furthermore statement, first note that $\left\|\sum \alpha_{n} e_{n}\right\| \geqslant\left\|\sum \alpha_{p(i)} e_{i}\right\|$ by the decreasing under sections. Now $\sum_{i}\left(\alpha_{p(i)}-\alpha_{p(i+1)}\right) e_{2 i}$ is the difference of $\sum \alpha_{p(i)}\left(e_{2 i-1}+e_{2 i}\right)$ by

$$
\alpha_{p(1)} e_{1}+\sum_{i \geqslant 2} \alpha_{p(i)}\left(e_{2 i-2}+e_{2 i-1}\right)
$$

which have the same norm as $\sum \alpha_{p(i)} e_{i}$ by repetition invariance.

REMARKS. 1. If $\|\cdot\|$ is a neighborly norm on $\left\{e_{n}\right\}$, then the $\rho$ constructed above need not be equivalent to $\|\cdot\|$. Indeed, in both the examples $1.3(\mathrm{c})$ and (d), the basic sequence $\left\{e_{2 n}\right\}_{n}$ is equivalent to the usual basis of $l_{2}$. However the two spaces are not isomorphic.

2. Neighborly bases have other interesting properties of which we mention one. If $\left\{e_{n}\right\}$ is neighborly, then $\left\{e_{2 n-1}-e_{2 n}\right\}_{n}$ is an unconditional basic sequence and $\left[e_{2 n-1}-e_{2 n}\right]_{n}$ is complemented in [ $\left.e_{n}\right]$. The dual of this statement is proved in [2]. The projection is $I-P$, where

$$
P\left(\sum \alpha_{n} e_{n}\right)=2^{-1} \sum_{n}\left(\alpha_{2 n-1}+\alpha_{2 n}\right)\left(e_{2 n-1}+e_{2 n}\right) .
$$

The norm of $P$ is one since it averages

$$
\sum_{n} \alpha_{2 n-1}\left(e_{2 n-1}+e_{2 n}\right) \text { and } \sum_{n} \alpha_{2 n}\left(e_{2 n-1}+e_{2 n}\right),
$$

both of which have norm $\leqslant\left\|\Sigma_{n} \alpha_{n} e_{n}\right\|$ by the neighborly property. The fact that $\left\{e_{2 n-1}-e_{2 n}\right\}$ is unconditional can be obtained by showing that this sequence is equivalent to $\left\{e_{2 n}\right\}$. The hard direction is illustrated by noting that

$$
\begin{gathered}
\alpha_{1} e_{1}-\alpha_{1} e_{2}+\alpha_{2} e_{3}-\alpha_{2} e_{4}, \\
\alpha_{1} e_{1}+\alpha_{1} e_{2}-\alpha_{1} e_{3}+\alpha_{2} e_{4}+\alpha_{2} e_{5}-\alpha_{2} e_{6}
\end{gathered}
$$

and

$$
\alpha_{1} e_{1}-\alpha_{1} e_{2}-\alpha_{1} e_{3}+\alpha_{2} e_{4}-\alpha_{2} e_{5}-\alpha_{2} e_{6}
$$

have the same norm by repetition invariance. Averaging the last two elements and using decreasing under sections yield that $\alpha_{1} e_{2}+\alpha_{2} e_{4}$ has a smaller norm.

3. There are quasireflexive spaces with no neighborly sequences (see Example 4.6).

The final topic of this section is duality. Let $\left\{e_{n}\right\}$ be a basis for $X$ and let $\left\{f_{n}\right\}$ be the coefficient functionals for $\left\{e_{n}\right\}$. The statement that $\left\{e_{n}\right\}$ is neighborly has a precise dual. Taking the transpose of the maps used to define neighborly, we find that $\left\{e_{n}\right\}$ is neighborly if and only if the $\left\{f_{n}\right\}$ satisfy the following condition:

(*) The norm of $\sum \beta_{n} f_{n}$ is not increased if either

(1) $\beta_{1}$ is replaced by zero, or

(2) $\beta_{k}$ is replaced by zero and $\beta_{k+1}$ is replaced by $\beta_{k}+\beta_{k+1}$, for $k \geqslant 1$, or

(3) $\beta_{k}$ is replaced by zero and $\beta_{k-1}$ is replaced by $\beta_{k-1}+\beta_{k}$, for $k \geqslant 2$.

It is also true that $\left\{f_{n}\right\}$ is neighborly if and only if $\left\{e_{n}\right\}$ satisfies $(*)$. If $\left\{f_{n}\right\}$ satisfies $(*)$, then it is easy to see that $\left\{f_{n}\right\}$ is invariant under spreading and decreasing under collapsing [14], that is,

$$
\left\|\sum \beta_{n} f_{n}\right\| \geqslant\left\|\sum_{i}\left(\sum_{n=n(i-1)+1}^{n(i)} \beta_{n}\right) f_{i}\right\|,
$$


for any increasing integer sequence $\{n(i)\}$ with $n(0)=0$. In [3], it is shown for real vector spaces, that if $\left\{f_{n}\right\}$ is linearly independent, then $\left\{f_{n}\right\}$ is invariant under spreading and decreasing under collapsing if and only if $\left\{f_{n}\right\}$ is ESA. Here ESA (equal signs additive) means that if $\beta_{k} \beta_{k+1} \geqslant 0$, then

$$
\left\|\sum \beta_{n} f_{n}\right\|=\left\|\sum_{n=1}^{k-1} \beta_{n} f_{n}+\left(\beta_{k}+\beta_{k+1}\right) f_{k}+\sum_{n=k+2}^{\infty} \beta_{n} f_{n}\right\| .
$$

It should be clear that invariant under spreading and decreasing under collapsing is equivalent to (2) and (3) of (*). With these comments we make the following definitions.

Definition 1.5. A sequence $\left\{f_{n}\right\}$ is said to be ESA (even for complex scalars) if it satisfies (2) and (3) of (*).

A sequence $\left\{f_{n}\right\}$ is dual neighborly if it satisfies (*).

If $\left\{f_{n}\right\}$ is ESA in this sense, then if it is renormed to be bimonotone, then in this new norm $\left\{f_{n}\right\}$ is dual neighborly. Note that a dual neighborly sequence is bimonotone due to the fact that it is dual to a neighborly sequence. We collect these results and a couple of straightforward facts in the next proposition.

Proposition 1.6. (a) If $\left\{e_{n}\right\}$ is a basis with coefficient functionals $\left\{f_{n}\right\}$, then $\left\{e_{n}\right\}$ is neighborly (resp. dual neighborly) if and only if $\left\{f_{n}\right\}$ is dual neighborly (resp. neighborly).

(b) If $\left\{e_{n}\right\}$ is neighborly and if $f_{n}=\sum_{i=1}^{n} e_{i}$, then $\left\{f_{n}\right\}$ is ESA.

(c) If $\left\{f_{n}\right\}$ is dual neighborly, then $\left\{f_{n}-f_{n+1}\right\}_{n}$ is neighborly.

(d) If $\left\{f_{n}\right\}$ is ESA, then $\left\{f_{n}\right\}$ is equivalent to a dual neighborly sequence.

REMARK. The $\left\{f_{n}\right\}$ in (b) need not be dual neighborly even if $\left\{e_{n}\right\}$ is the basis for $J$ in Example 1.3(c). Indeed, in general the Projection Principle only yields that the map in (1) of $(*)$ has norm $\leqslant 2$ in (b).

If $\left(\left\{e_{n}\right\},\|\cdot\|\right)$ is invariant under spreading, then the norm $\|\cdot\|$ can be extended in an obvious way to the collection of all finitely nonzero scalar-valued functions on any linear ordered set $L$. The completion of this space is also a space of functions on $L$.

2. Transfinite duals. The notation in this section could easily get out of hand. The reader is warned that there are several different orderings around. We will adopt the following conventions:

(1) $L=I \times Z$, where $I=[0,1]$ and $Z$ is the set of all integers.

(2) $L$ is linearly ordered by the lexicographical ordering, namely $\left(i_{1}, z_{1}\right)<\left(i_{2}, z_{2}\right)$ if $i_{1}<i_{2}$ or if $i_{1}=i_{2}$ and $z_{1}<z_{2}$. If there is a chance of confusion this ordering will be denoted by $<_{L}$.

(3) If $\lambda \in L$, we will denote by $e_{\lambda}$ the function on $L$ which is one at $\lambda$ and zero otherwise.

(4) For each positive integer $n$ for which $n=2^{k}+j$ with $0 \leqslant j<2^{k}$ and $k \geqslant 0$, we denote by $\theta_{n}$ the function on $L$ which is one on each $\lambda$ satisfying $\left(j / 2^{k}, 1\right) \leqslant \lambda \leqslant$ $\left((j+1) / 2^{k},-1\right)$ and zero otherwise. 
(5) For each positive integer $k$, let $A_{k}=\left\{j / 2^{k}: 0<j<2^{k}\right\}$ and let $D_{k}=A_{k} \backslash A_{k-1}$ for $k \geqslant 2$ and $D_{1}=A_{1}=\{1 / 2\}$. We will order $D=\cup_{k=1}^{\infty} D_{k}$ by giving each $D_{k}$ its usual ordering and requiring that if $x \in D_{k}, y \in D_{j}$ with $k \neq j$, then $x<y$ if and only if $k<j$. If there is a chance of confusion this ordering will be denoted by $<_{D}$.

(6) We will assume $\left\{e_{n}\right\}$ is a neighborly basis and hence $\left\{f_{n}\right\}$, the set of its coefficient functionals, is a dual neighborly basic sequence.

(7) Denote by $B$ the set

$$
\left\{\theta_{n}\right\}_{n=1}^{\infty} \cup\left\{e_{(x, i)}: x \in D, i \in Z \backslash\{0\}\right\} \cup\left\{e_{(0, i)}\right\}_{i=1}^{\infty} \cup\left\{e_{(1,-i)}\right\}_{i=1}^{\infty} .
$$

Let $E_{0}$ be the span of $B$ and let $E$ be the closure of $E_{0}$ in the norm obtained by extending the neighborly norm on $\left[e_{n}\right]$ to the space of functions on $L$.

(8) Denote by $B^{\prime}$ the set

$$
\left\{e_{(x, i)}: x \in D, i \in Z\right\} \cup\left\{e_{(0, i)}\right\}_{i=1}^{\infty} \cup\left\{e_{(1,-i)}\right\}_{i=1}^{\infty}
$$

and let $C=B^{\prime} \cup\left\{e_{(x, 0)}: x \in I \backslash D\right\}$. Let $F_{0}$ be the span of $C$ and let $F$ be the closure of $F_{0}$ in the norm obtained by extending the ESA norm on $\left[f_{n}\right]$ to the space of functions on $L$.

(9) Order the set $B$ by $<_{B}$ in the following order: $\theta_{n}<\theta_{m}$, if $n<m ; \theta_{n}<e_{(x, i)}$ for each $e_{(x, i)} \in B$; $e_{(x, i)}<e_{(y, j)}$ if $|i|<|j|$, or if $|i|=|j|$ and $i<j$, or if $i=j$ and $x$, $y \in D$ and $x<_{D} y$, or if $i=j$ and $x=0$ or 1 and $y \in D$.

We collect a couple of straightforward observations in the next lemma.

LEMMA 2.1. (i) If $\left\{e_{n}\right\}$ is a shrinking basis for $X$ and if $Y$ is a subspace of $X^{\prime}$ such that for each $n$ there is $f_{n} \in Y$ with $f_{n}\left(e_{m}\right)=\delta_{n m}$, then $Y$ is dense in $X^{\prime}$.

(ii) Suppose $M$ and $N(1), \ldots, N(m)$ are pairwise disjoint sets whose union is the set of positive integers, and suppose $M$ is finite and each $N(j), 1 \leqslant j \leqslant m$, is infinite. Let $\left\{x_{n}\right\}$ be a basic sequence. Then

(a) if $\left\{x_{n}: n \in N(j)\right\}$ is shrinking for $1 \leqslant j \leqslant m$ then $\left\{x_{n}\right\}$ is shrinking;

(b) if $\left[x_{n}: n \in N(j)\right]^{2}=\left[\left\{\sum_{n \in N(j)} x_{n}\right\} \cup\left\{x_{n}: n \in N(j)\right\}\right]$ then $\left[x_{n}\right]^{2}=\left[\left\{x_{n}\right\} \cup\right.$ $\left.\left\{\sum_{n \in N(j)} x_{n}\right\}_{j=1}^{m}\right]$.

LEMMA 2.2. With the above conventions, we have:

(i) The map $\langle\cdot, \cdot\rangle: E_{0} \times F_{0} \rightarrow$ scalars given by

$$
\langle g, h\rangle=\sum_{\lambda \in L} g(\lambda) h(\lambda)
$$

extends to a function on $E \times F$ which makes $(E, F)$ into a dual pair. Furthermore, with this pairing, each of $E$ and $F$ norms the other.

(ii) For each scalar-valued finitely nonzero function $f$ on $B$ and each $\beta \in B$,

$$
\left\|\sum_{b \in B} f(b) b\right\| \geqslant\left\|\sum_{b \leqslant \beta} f(b) b\right\| \text {. }
$$

Hence each subsequence of $B$ (i.e. a co-final sequence in $\left(b,<_{B}\right)$ ) is a monotone basic sequence in $E$.

(iii) If both $\left\{e_{n}\right\}$ and its inversion are shrinking (respectively, span a quasireflexive space) and $\left\{x_{n}\right\}$ is a subsequence of $B$ which has the property that, if $e_{(y, i)} \in\left\{x_{n}\right\}$, $|j|<|i|$ and $e_{(y, j)} \in B$, then $e_{(y, j)} \in\left\{x_{n}\right\}$, then $\left\{x_{n}\right\}$ is shrinking (respectively, spans a quasireflexive space). 
Proof. Since $|\langle g, h\rangle| \leqslant\|g\|\|h\|$, if $(g, h) \in E_{0} \times F_{0}$ and each of $E_{0}$ and $F_{0}$ norms the other, we have (i).

The proof of (ii) follows from the Projection Principle and the ordering on $B$.

To see (iii), note that the condition on $\left\{x_{n}\right\}$ implies that there is a finite subset $H$ of $D \cup\{0,1\}$ so that $b \in\left\{x_{n}\right\}$ implies that $b=e_{(d, i)}$ for some $d \in H$, with at most a finite number of exceptions. Hence the result follows from Lemma 2.1(ii).

For each $\alpha \leqslant \omega$ define a set $A_{\alpha}$ by

$$
\begin{aligned}
& A_{0}=A_{1}=\left\{e_{(0, i)}\right\}_{i=1}^{\infty} ; \\
& A_{2 m+1}=A_{1} \cup\left\{e_{(1,-i)}\right\}_{i=1}^{m}, \quad \text { for } m \geqslant 1 ; \\
& A_{2 m+2}=A_{2 m+1} \cup\left\{\theta_{1}\right\}, \quad \text { for } m \geqslant 0 ; \\
& A_{\omega}=\bigcup_{n} A_{2 n} .
\end{aligned}
$$

Let $H_{\omega}=\cup_{n} A_{2 n+1}$.

THEOREM 2.3. With the conventions of this section and if $\left[e_{n}\right]$ is quasireflexive and $\alpha \leqslant \omega$, then $\left[e_{n}\right]$ is isometrically isomorphic to the closed linear span of $A_{\alpha}$ in $E$, if $\alpha$ is even, or in $F$, if $\alpha$ is odd. Furthermore $\left[f_{n}\right]^{\omega}$ is isometrically isomorphic to the closed linear span of $H_{\omega}$ in $F$.

Proof. This is obvious for $\alpha=0$ and $\alpha=1$. The two results with $\alpha=\omega$ will follow from the results with $\alpha<\omega$, provided we show that the inclusion maps: $\left[A_{\alpha}\right] \rightarrow\left[A_{\alpha+2}\right]$ are the canonical injections: $\left[e_{n}\right]^{\alpha} \rightarrow\left[e_{n}\right]^{\alpha+2}$. We complete the proof by induction, separating the cases into $\alpha$ even or $\alpha$ odd.

Assume the result is true for $\alpha=2 m$ and $\alpha=2 m+1$. Since $A_{2 m} \subset B$, by Lemma 2.2, $\left(A_{2 m},<_{B}\right)$ is a monotone shrinking basis for $\left[e_{n}\right]^{2 m}$. From Lemma 2.1(ii) we have $\left[e_{n}\right]^{2 m+2}$ is isometrically isomorphic to $\left[A_{2 m} \cup\left\{\sum_{i=1}^{\infty} e_{(0, i)}\right\}\right]$. Let $\mu \in L$ and let $\phi_{\mu}$ be the function on $L$ which is one at each $\lambda$ with $(0,1) \leqslant \lambda \leqslant \mu$, and zero otherwise. If $\mu>(0, i)$ for each $i$ and if $\mu \leqslant(1,-m-1)$, then the space $\left[A_{2 m} \cup\right.$ $\left.\left\{\sum_{i=1}^{\infty} e_{(0, i)}\right\}\right]$ is isometrically isomorphic, by the obvious map, to [ $\left.A_{2 m} \cup\left\{\phi_{\mu}\right\}\right]$. The map $\left[A_{2 m}\right] \rightarrow\left[A_{2 m} \cup\left\{\phi_{\mu}\right\}\right]$ will be the canonical injection provided $\left\langle e_{(1, j)}, \phi_{\mu}\right\rangle=0$ for each $e_{(1, j)} \in A_{2 m+1}$, that is if $\mu \leqslant(1,-m-1)$. Hence, when $\mu=(1,-m-1)$, we add $\phi_{\mu}$ to $A_{2 m}$ to obtain a set with the properties of $A_{2 m+2}$. If $m=0$, then $\phi=\theta_{1}$ and we have constructed $A_{2}$. If $m>0$, note that $\phi=\theta_{1}-\sum_{i=1}^{m} e_{(1,-i)}$ and $e_{(1,-m)}=$ $\theta_{1}-\phi-\sum_{i=1}^{m-1} e_{(1,-i)}$. Thus $\left[A_{2 m+2}\right]=\left[A_{2 m} \cup\{\phi\}\right]$ and the result is true for $\alpha=$ $2 m+2$.

Assume the result is true for $\alpha=2 m$ and $\alpha=2 m-1$, and let $Y$ be the span of $A_{2 m+1}$. The hypothesis of Lemma 2.1(i) is satisfied. Hence $Y$ as a set is dense in $\left[e_{n}\right]^{2 m+1}$. Each element in $Y$ has two norms, $\|\cdot\|_{F}$ which $Y$ inherits as a subspace of $F$, and $\|\cdot\|^{\prime}$ as a functional on $\left[e_{n}\right]^{2 m}$. Since $(E, F)$ is a norming dual pair, we have $\|\cdot\|^{\prime} \leqslant\|\cdot\|_{F}$ on $Y$. To show $\left[e_{n}\right]^{2 m+1}$ is isometrically isomorphic to $\left[A_{2 m+1}\right]$, it suffices to show these norms are equal. This would complete the proof, since the inclusion $\left[A_{2 m-1}\right] \rightarrow\left[A_{2 m+1}\right]$ would be the canonical injection.

To see that these norms are equal let $y \in Y$ be given by $y=\sum_{i=1}^{k} a_{i} e_{(0, i)}+$ $\sum_{i=-m}^{-1} b_{i} e_{(1, i)}$. Now $\|y\|_{F}=\left\|\sum_{i=1}^{k} a_{i} f_{i}+\sum_{i=-m}^{-1} b_{i} f_{k+m+1-i}\right\|$ and hence there are 
$\left\{c_{i}\right\}_{1}^{k}$ and $\left\{d_{i}\right\}_{-m}^{-1}$ so that $\left\|\sum_{i=1}^{k} c_{i} e_{i}+\sum_{i=-m}^{-1} d_{i} e_{k+m+1-i}\right\|=1$ and $\sum_{1}^{k} a_{i} c_{i}+\sum_{-m}^{-1} b_{i} d_{i}$ $=\|y\|_{F}$. Consider the element $x$ in $\left[A_{2 m}\right]$ given by

$$
x=d_{-m} \theta_{1}+\sum_{i=1}^{k}\left(c_{i}-d_{-m}\right) e_{(0, i)}+\sum_{i=-m+1}^{-1}\left(d_{i}-d_{-m}\right) e_{(1, i)} .
$$

We have $\|x\|=1$ and $\langle y, x\rangle=\|y\|_{F}$. Hence the two norms agree and the proof is complete.

COROLlARY 2.4. If $\left\{x_{n}\right\}$ is a neighborly or ESA basis for a quasireflexive space, then $\left[x_{n}\right]^{\omega}$ is isomorphic to $\left[x_{n}\right] \oplus\left[y_{n}\right]$, where $\left\{y_{n}\right\}$ is the inversion of $\left\{x_{n}\right\}$.

We continue to define the sets $A_{\alpha}$ for $\alpha \leqslant \omega^{2}$ by

$$
\begin{aligned}
& A_{n \omega}=A_{\omega} \cup\left\{\theta_{i}\right\}_{i=1}^{2^{n}-1} \cup\left\{e_{(x, i)}: i \in Z \backslash\{0\}, x \in D_{k}, k \leqslant n-1\right\} ; \\
& A_{n \omega+2}=A_{n \omega} \cup\left\{\theta_{i}\right\}_{i=1}^{2^{n+1}-1} ; \\
& A_{n \omega+2 m+2}=A_{n \omega+2} \cup\left\{e_{(x, i)}: i= \pm 1, \ldots, \pm m, x \in D_{n}\right\} ; \\
& A_{n \omega+1}=H_{\omega} \cup\left\{e_{(x, i)}: i \in Z, x \in D_{k}, k \leqslant n-1\right\} \cup\left\{e_{(x, 0)}: x \in D_{n}\right\} ; \\
& A_{n \omega+2 m+1}=A_{n \omega+1} \cup\left\{e_{(x, i)}: x \in D_{n},|i| \leqslant m\right\} ; \\
& A_{\omega^{2}}=\cup\left\{A_{\alpha}: \alpha \text { even }\right\} .
\end{aligned}
$$

We also define sets $H_{\alpha}$ for $\omega<\alpha \leqslant \omega^{2}$ by

$$
\begin{aligned}
& H_{n \omega}=H_{\omega} \cup\left\{e_{(x, i)}: i \in Z \backslash\{0\}, x \in D_{k}, k \leqslant n-1\right\} ; \\
& H_{n \omega+1}=H_{n \omega} \cup\left\{\theta_{i}\right\}_{i=2^{n}}^{2^{n+1}-1} ; \\
& H_{n \omega+2 m}=H_{n \omega} \cup\left\{e_{(x, i)}: x \in D_{n}, i= \pm i, \ldots, \pm m\right\} ; \\
& H_{n \omega+2 m+1}=H_{n \omega+1} \cup\left\{e_{(x, i)}: x \in D_{n}, i= \pm 1, \ldots, \pm m\right\}, \\
& H_{\omega^{2}}=\cup\left\{H_{\alpha}: \alpha \text { even, } \alpha \geqslant \omega\right\} .
\end{aligned}
$$

THEOREM 2.5. With the conventions of this section and if both $\left\{e_{n}\right\}$ and its inversion span a quasireflexive space and if $\alpha \leqslant \omega^{2}$, then $\left[e_{n}\right]^{\alpha}$ is isometrically isomorphic to the closed linear span of $A_{\alpha}$ in $E$ if $\alpha$ is even, and in $F$ is $\alpha$ is odd. Furthermore, if $\omega \leqslant \alpha \leqslant \omega^{2}$, then $\left[f_{n}\right]^{\alpha}$ is isometrically isomorphic with the closed linear span of $H_{\alpha}$ in $F$ if $\alpha$ is even, and in $E$ if $\alpha$ is odd.

Proof. Again limit ordinals are no problem provided the map $\left[A_{\alpha}\right] \rightarrow\left[A_{\alpha+2}\right]$ is the canonical injection $\left[e_{n}\right]^{\alpha} \rightarrow\left[e_{n}\right]^{\alpha+2}$. Assume the result is true for $\alpha$.

If $\alpha+1$ is odd, the proof given in Theorem 2.3 generalizes. The only difference, besides the increase in notation, is how to choose $x$. Pick the coefficients of the $\left\{\theta_{i}\right\}$ 's first, in that order, to give $x$ the correct values at the points $\{(z, 0): z \in D\}$. Then the other choices are fixed.

If $\alpha+1$ is even, the proof given in Theorem 2.3 generalizes. At each stage, we replace elements of the form $\sum_{i=1}^{\infty} e_{(d, i)}$ or $\sum_{i=1}^{\infty} e_{(d,-i)}$ with the longest characteristic function of an interval in $L$ so that we preserve both the isometry and the canonical injection. 
The proof of the furthermore statement follows in a similar manner, once we have noticed that $\left[H_{n \omega}\right]$ in $F$ is isometrically the dual of $\left[H_{n \omega}\right]$ in $E$.

COROllary 2.6. If $\left\{x_{n}\right\}$ is a neighborly or ESA basis and both $\left\{x_{n}\right\}$ and its inversion span quasireflexive spaces, then $\left[x_{n}\right]^{k \omega+m}$ is quasireflexive of order $2^{k},\left[x_{n}\right]^{\omega^{2}}$ is separable and $\left[x_{n}\right]^{\omega^{2}+1}$ is not separable.

Proof. Since $A_{k \omega+n+2}$ has $2^{k}$ more elements than $A_{k \omega+n},\left[e_{n}\right]^{k \omega+n}$ is quasireflexive of order $2^{k}$. Since $A_{\omega^{2}}$ is countable, $\left[e_{n}\right]^{\omega^{2}}$ is separable. To see $\left[e_{n}\right]^{\omega^{2}+1}$ is nonseparable, consider the uncountable set $\left\{e_{(x, 0)}: x \in I \backslash D\right\} \subset F$. Each is a continuous linear functional on $\left[e_{n}\right]^{\omega^{2}}$, and if $x<y$ and $j / 2^{k} \in D$ is the first element in $\left(D,<_{D}\right)$ with $x<j / 2^{k}<y$, then $\left\langle e_{(x, 0)}, \theta_{2^{k}+j}\right\rangle=0$ and $\left\langle e_{(y, 0)}, \theta_{2^{k}+j}\right\rangle=1$. Since all the $\theta_{n}$ 's have the same norm, $\left[e_{n}\right]^{\omega^{2}+1}$ is nonseparable.

COROllaRY 2.7. (i) If $\left\{e_{n}\right\}$ is a neighborly or ESA basis, then either $\left[e_{n}\right]^{2}$ is nonseparable or $\left[e_{n}\right]^{\alpha}$ is separable for $\alpha \leqslant \omega$; either $\left[e_{n}\right]^{\omega+2}$ is nonseparable or $\left[e_{n}\right]^{\alpha}$ is separable for $\alpha \leqslant \omega^{2}$.

(ii) If $X$ is quasireflexive of order one, then either $X^{\omega+2}$ is nonseparable or $X^{\alpha}$ is separable for $\alpha \leqslant 2 \omega$; either $X^{2 \omega+2}$ is nonseparable or $X^{\alpha}$ is separable for $\alpha \leqslant \omega^{2}$.

(iii) If $X$ is nonreflexive, then $X^{\omega^{2}+1}$ is nonseparable.

Proof. (i) $\left[e_{n}\right]^{2}$ (respectively, $\left[e_{n}\right]^{\omega+2}$ ) separable implies that $\left\{e_{n}\right\}$ (respectively, its inversion) spans a quasireflexive space.

(ii) Perrot [14] shows that $X^{\omega}$ is isomorphic to $X \oplus\left[x_{n}\right]$ where $\left\{x_{n}\right\}$ is a ESA sequence. Since $X^{k \omega}$ is then isomorphic to $X \oplus\left[x_{n}\right]^{\omega} \oplus\left[x_{n}\right]^{2 \omega} \oplus \cdots \oplus\left[x_{n}\right]^{(k-1) \omega}$, the result follows from (i).

(iii) Perrott [14] shows that $X^{\omega}$ contains an ESA sequence $\left\{x_{n}\right\}$ and since $\left[x_{n}\right]^{\omega^{2}} \subset\left(X^{\omega}\right)^{\omega^{2}}=X^{\omega^{2}}, X^{\omega^{2}+1}$ is nonseparable by (i).

REMARKS. 1. In [5], it is shown that if $X$ is nonreflexive then $X^{\omega^{2}+2}$ is nonseparable.

2. For a space $X$, define $s(X)=\min \left\{\alpha: X^{\alpha}\right.$ is nonseparable $\}$. Corollary 2.7 tells us that if $X$ is separable and quasireflexive then $s(X) \in\{\omega+1, \omega+2,2 \omega+1,2 \omega+$ $\left.2, \omega^{2}+1\right\}$ and if $\left\{e_{n}\right\}$ is a neighborly basis spanning a quasireflexive space then $s\left(\left[e_{n}\right]\right) \in\left\{\omega+1, \omega+2, \omega^{2}+1\right\}$. The examples in $\$ 4$ will show that each of these ordinals is a possible value for $s(X)$. Thus we have $\{1,2, \ldots, \omega+1, \omega+2,2 \omega+$ $\left.1,2 \omega+2, \omega^{2}+1\right\} \subset\{s(X): X$ is separable and nonreflexive $\} \subset\left\{\alpha \leqslant \omega^{2}+1: \alpha\right.$ is not a limit ordinal $\}$. The existence of spaces $X$ with $X^{n}$ separable, but $X^{n+1}$ nonseparable was given in [6].

3. The method used to obtain $\left[e_{n}\right]^{\omega^{2}}$ stops there. Indeed since $\left[e_{n}\right]^{\omega^{2}+1}$ is nonseparable, $\left[e_{n}\right]^{\omega^{2}}$ cannot have a shrinking basis.

The next step is to consider the space $\left[\theta_{n}\right]$ and some of its nice subspaces. We need to know yet another ordering. The tree ordering, denoted by $<_{T}$, on the set of positive integers is the partial ordering obtained by using transitivity to the relations $n<_{T} 2 n$ and $n<_{T} 2 n+1$. Note that the elements $\left\{n: 2^{k} \leqslant n<2^{k+1}\right\}$ are incompatible in this ordering and $n<_{T} m$ if and only if the support of $\theta_{n}$ contains the support of $\theta_{m}$. A branch is a maximal linearly ordered subset of $\left(N,<_{T}\right)$. There are 
uncountably many branches. Let $S$ be the set of odd positive integers and let $P$ be the set of even positive integers. (The letters $P$ and $S$ are suggested by port and starboard, i.e., left or right.) We divide the set of the branches into three sets:
$\mathscr{P}$ the set of branches $Q$ so that $Q \cap S$ is finite,
$\delta$ the set of branches $Q$ so that $Q \cap P$ is finite, and
9 the set of branches $Q$ so that $Q \notin \mathscr{P} \cup \mathcal{S}$.

We note that $\mathcal{P}$ and $\mathcal{S}$ are countable sets and $\mathcal{G}$ is uncountable.

THEOREM 2.8. With the conventions of this section, and if $\left\{e_{n}\right\}$ and its inversion $\left\{x_{n}\right\}$ are quasireflexive, then:

(i) The space $\left[\theta_{n}\right]$ is norm one complemented in $\left[e_{n}\right]^{\omega^{2}}$ and the two spaces are isomorphic.

(ii) Let $Q$ be a branch. If $Q \in \mathcal{P}$, then $\left[\theta_{n}: n \in Q\right]$ is isomorphic to $\left[x_{n}\right]$; if $Q \in \mathcal{S}$, then $\left[\theta_{n}: n \in Q\right]$ is isomorphic to $\left[e_{n}\right]$; and if $Q \in \mathcal{G}$, then $\left[\theta_{n}: n \in Q\right]$ is isomorphic to $\left[e_{n}\right] \oplus\left[x_{n}\right]$ and $\left\{\theta_{n}: n \in Q\right\}$ is neither shrinking nor boundedly complete.

Proof. The Projection Principle yields a norm one projection onto $\left[\theta_{n}\right]$ with kernel the closed linear span in $E$ of the set $K$ where

$$
K=\left\{e_{(d, i)}: i \in Z \backslash\{0\}, d \in D \cup\{0,1\} \cap E\right\} .
$$

Furthermore, if $Q$ is the branch $\left\{2^{k}: k=0,1, \ldots\right\}$ and $Y=\left[\theta_{n}: n \in Q\right]$, then the Projection Principle yields a projection $U:\left[\theta_{n}\right] \rightarrow Y$. The map $V: Y \rightarrow Y$ given by $V\left(\Sigma_{n \in Q} \alpha_{n} \theta_{n}\right)=\Sigma_{n \in Q} \alpha_{n} \theta_{2 n}$ is an isometry. Thus the map $I-U+V U$ is an isomorphism of $\left[\theta_{n}\right]$ onto $\left[\theta_{n}\right]_{n=2}^{\infty}$ and hence $\left[\theta_{n}\right]$ is isomorphic to its own square.

The space $\left[e_{n}\right]^{\omega^{2}}$ is also isomorphic to its own square. Indeed $\left[e_{n}\right]^{2 \omega}$ is isomorphic to $\left[e_{n}\right]^{\omega} \oplus\left[e_{n}\right]^{\omega}$ by Corollary 2.4 . Hence $\left[e_{n}\right]^{\omega^{2}}$ is isomorphic to

$$
\left(\left[e_{n}\right]^{2 \omega}\right)^{\omega^{2}}, \quad\left(\left[e_{n}\right]^{\omega} \oplus\left[e_{n}\right]^{\omega}\right)^{\omega^{2}}, \quad\left(\left[e_{n}\right]^{\omega}\right)^{\omega^{2}} \oplus\left(\left[e_{n}\right]^{\omega}\right)^{\omega^{2}} \text { and }\left[e_{n}\right]^{\omega^{2}} \oplus\left[e_{n}\right]^{\omega^{2}} \text {. }
$$

Therefore, to show $\left[\theta_{n}\right]$ and $\left[e_{n}\right]^{\omega^{2}}$, it suffices, by the Pelczynski decomposition method [12, p. 54] to show that the closed linear span of $K$ is isomorphic to a complemented subspace of $\left[\theta_{n}\right]$.

Let $K_{\infty}$ be the set $K$ together with the functions $\sum_{i=1}^{\infty} e_{(d, i)}, d \in D \cup\{0\}$ and $\sum_{i=1}^{\infty} e_{(d,-i)}, d \in D \cup\{1\}$. The claim is that the span of $K$ and the span of $K_{\infty}$ are isomorphic. The proof of this is like the proof of Proposition 1.2(e) only done simultaneously for all $d \in D \cup\{1,0\}$. The set

$$
K_{0}=\left\{\sum_{i=n}^{\infty} e_{(d, i)}: d \in D \cup\{0\}, n \geqslant 1\right\} \cup\left\{\sum_{i=n}^{\infty} e_{(d,-i)}: d \in D \cup\{1\}, n \geqslant 1\right\}
$$

has the same span as $K_{\infty}$. Note that $\left\{\sum_{i=n}^{\infty} e_{(d, i)}\right\}$ and $\left\{\theta_{n}: n \in Q, n \geqslant m\right\}$ are 1 -equivalent basic sequences if the set $\{n \in Q, n \geqslant m\} \subset S$. A similar statement is true for $\left\{\sum_{i=n}^{\infty} e_{(d,-i)}\right\}$ and branches whose tail-end is contained in $P$.

An induction argument shows there is a set of branches

$$
\mathscr{B}=\{Q(d, \varepsilon): d \in D, \varepsilon= \pm 1 \text { or } d=0, \varepsilon=1 \text { or } d=1, \varepsilon=-1\}
$$


and an integer-valued function $f$ on $(D \cup\{0,1\}) \times\{-1,1\}$ :

(i) If $\varepsilon=1$ (respectively, -1) then $T(d, \varepsilon)=\{n \in Q(d, \varepsilon): n \geqslant f(d, \varepsilon)\}$ is contained in $S$ (respectively $P$ ).

(ii) If $d_{1}<d_{2}$ as real numbers or if $d_{1}=d_{2}$ and $\varepsilon_{1}=-1$ and $\varepsilon_{2}=1$, then for each $i \in T\left(d_{1}, \varepsilon_{1}\right)$ and $j \in T\left(d_{2}, \varepsilon_{2}\right)$ and each $\lambda, \mu$ in $L$ with $\theta_{i}(\lambda)=1$ and $\theta_{j}(\mu)=1$, it follows that $\lambda<_{L} \mu$. (The "supports" of $T(d, \varepsilon)$ are in the same $L$-order as things in $K_{0}$.)

Let $M=\cup \mathscr{B}, W=\cup\{T(d, \varepsilon): Q(d, \varepsilon) \in \mathscr{B}\}$. We have that $\left[K_{0}\right]$ is isometric with $\left[\theta_{n}: n \in W\right]$, and the Projection Principle yields projections onto [ $\left.\theta_{n}: n \in M\right]$ and $\left[\theta_{n}: n \in M \backslash W\right]$ whose difference is a projection onto $\left[\theta_{n}: n \in W\right]$. This completes the proof of (i).

Suppose $Q$ is a branch in $\mathcal{S}$ (respectively, $\mathscr{P}$ ) then $\left[\theta_{n}: n \in Q, n \geqslant m\right]$ is isomorphic to $\left[e_{n}\right]$ (respectively, $\left.\left[x_{n}\right]\right)$ as before. Thus the same is true about $\left[\theta_{n}: n \in Q\right]$ by Proposition 1.2(f). The basic sequence $\left\{\theta_{n}: n \in Q\right\}$ is not shrinking for any branch $Q$ since the functional $\Sigma_{n \in Q} \alpha_{n} \theta_{n} \rightarrow \Sigma_{n \in Q} \alpha_{n}$ is continuous.

Now let $Q \in 9$. Inductively choose $\{n(i)\} \subset Q$ so that $n(1)>1$ and either $n(i+1)+1 \in P$ and $j \in S$ for $n(i)<j \leqslant n(i+1)$, or $n(i+1)+1 \in S$ and $j \in P$ for $n(i)<j \leqslant n(i+1)$. Each sum $\sum_{i=1}^{k}(-1)^{i+1} \theta_{n(i)}$ is the characteristic function of an interval in $L$ and hence $\left\{\theta_{n}: n \in Q\right\}$ is not boundedly complete. Let $\{m(i)\}$ be a listing of $Q$, in order. Consider the basis $\left\{x_{n}\right\}$ of $\left[\theta_{n}: n \in Q\right]$ given by $x_{1}=\theta_{m(1)}$ and $x_{i+1}=\theta_{m(i)}-\theta_{m(i+1)}$, for $i \geqslant 1$. It can be checked that $\left\{y_{i+1}, m(i+1) \in S, i \geqslant 1\right\}$ (respectively $\left\{y_{i+1}, m(i+1) \in P, i \geqslant 1\right\}$ ) is 1-equivalent to $\left\{e_{n}\right\}$ (respectively $\left\{x_{n}\right\}$ ). Hence the proof is complete.

3. Function spaces. This section shows the relationships between $J^{\omega^{2}}$ and $J F$, James function space. The results are more general and are so stated and proved.

Throughout this section $\left(\left\{x_{n}\right\},\|\cdot\|\right)$ will be a neighborly basis and norm for the Banach space $X$. The sequence $\left\{y_{n}\right\}$ will be the ESA basis for $Y$, its closed linear span, where $y_{n}=\sum_{i=n}^{\infty} x_{i}$.

We concern ourselves with three function spaces on $I$. The norm in the first two spaces is the neighborly norm on $\left\{x_{n}\right\}$ extended to the space of functions on $I$ as in $\S 1$.

(1) $X W 2$ : the closure in the neighborly norm of the linear span of the characteristic functions $\chi_{\left(j / 2^{k},(j+1) / 2^{k}\right)}$ for $0 \leqslant j<2^{k}, k \geqslant 0, j$ and $k$ integers. If $\left\{x_{n}\right\}$ and its inversion both span quasireflexive spaces, then $X W 2$ is isomorphic to the $\omega^{2}$-dual of $X$ by Theorem 2.8 .

(2) $X F$ : the closure in the neighborly norm of the space of all continuous piecewise linear functions on $I$. It is easy to check that functions in $X F$ are continuous.

(3) $Y F$ : the completion of the span of all characteristic functions of intervals contained in $I$ with norm defined by

$$
\|f\|=\sup \left\{\left\|\sum_{i=1}^{n}\left(\int_{t(i-1)}^{t(i)} f\right) y_{i}\right\|: 0=t(0)<t(1)<\cdots<t(n)=1\right\} \text {. }
$$

This space was constructed in [5]. 
LEMMA 3.1. The map $f(t) \rightarrow \int_{0}^{t} f(s) d s$ is an isometry from $Y F$ onto the codimension one subspace of $X F$ of all functions $g \in X F$ with $g(0)=0$.

Proof. Clearly this map is a correspondence between step functions in $Y F$ and continuous piecewise linear functions $g \in X F$ with $g(0)=0$. Thus it suffices to show that the norms are the same.

$$
\begin{aligned}
& \text { If } g(t)=\int_{0}^{t} f(s) d s \text { and } 0=t(0)<t(1)<\cdots<t(n)=1, \text { then } \\
& \qquad \begin{aligned}
\sum_{i=1}^{n}\left(\int_{t(i-1)}^{t(i)} f\right) y_{i} & =\sum_{i=1}^{n}(g(t(i))-g(t(i-1))) y_{i} \\
& =-g(0) y_{i}+\sum_{i=1}^{n-1} g(t(i))\left(y_{i}-y_{i+1}\right)+g(t(n)) y_{n} \\
& =\sum_{i=1}^{n-1} g(t(i)) x_{i}+g(t(n)) \sum_{i=n}^{\infty} x_{n} .
\end{aligned}
\end{aligned}
$$

Since this last element has the same norm as $\sum_{i=1}^{n} g(t(i)) x_{i}$, taking sups shows that the norms agree. This completes the lemma.

We use the Haar system and Schauder system as defined in [12, p. 3]. Note that the map in Lemma 3.1 sends the $n$th element of the Haar system to the $n+1$ st element of the Schauder system.

LEMMA 3.2. The Haar system is a monotone basis for YF and the Schauder system is a monotone basis for $X F$.

PROOF. The characteristic functions of intervals with dyadic endpoints have dense linear span in $Y F$. If $f \in Y F$ is a step function on $I$ whose points of discontinuity are contained in $\{t(i)\}_{i=0}^{n}$ with $0=t(0)<t(1)<\cdots<t(n)=1$, then, since $\left\{y_{n}\right\}$ is ESA, $\|f\|=\left\|\sum_{i=1}^{n}\left(\int_{t(i-1)}^{t(i)} f\right) y_{i}\right\|$. Thus if $g$ is any function with $\int_{t(i-1)}^{t(i)} g=0$ for $i=1,2, \ldots, n$, then $\|f+g\| \geqslant\|f\|$. Hence the Haar system is monotone basis for $Y F$.

If $f$ is a continuous piecewise linear function and if $0=t(0)<t(1)<\cdots<t(n)$ $=1$ are such that $f$ is linear on each interval $(t(i-1), t(i))$, then by Lemma 1.1(c), $\|f\|=\left\|\sum_{i=0}^{n} f(t(i)) x_{i}\right\|$. Thus if $g$ is a function in $X F$ with $g(t(i))=0$ for $i=$ $1,2, \ldots, n$, then $\|f+g\| \geqslant\|f\|$. Hence the Schauder system is a monotone basis for $X F$.

REMARKs. 1. It is not hard to show that $Y F$ is isomorphic to $Y F \oplus Y F$. The author does not know if $X F$ is isomorphic to $X F \oplus X F$. If not this would be an example of a space not isomorphic to a codimension one subspace.

2. James function space [11] is isomorphic to $Y F$ and hence to a codimension one subspace of $X F$, when $X=J$ (Example 1.3(c)).

THEOREM 3.3. Suppose $\left\{x_{2 n}\right\}_{n}$ is not equivalent to the usual basis of $l_{1}$. Then XF is a subspace of $X W 2$ and $X W 2$ is isometric to a complemented subspace of $X F$.

Proof. Let $\lambda(n)=\left\|\sum_{i=1}^{n} x_{2 i}\right\|$. The condition on $\left\{x_{2 n}\right\}_{n}$ is equivalent to $\lim \lambda(n) / n=0[12$, p. 120]. Let $g \in X F$ be continuous and let $g$ be linear on each of the intervals $\left[j / 2^{m},(j+1) / 2^{m}\right]$ for $0 \leqslant j<2^{m}$ and for some $m \geqslant 0$. If necessary, 
redefine $g(0)=g(1)=0$. This does not change the norm of $g$. Let $M$ be the largest slope in absolute value of any of the linear pieces of $g$.

For $n=2^{k}+j$, with $0 \leqslant j<2^{k}$ and $k \geqslant 0$, define $\theta_{n}=\chi_{\left(j / 2^{k},(j+1) / 2^{k}\right)}$. Inductively choose scalars $\left\{\beta_{n}\right\}$ so that the functions $g$ and $g_{n}=\sum_{i=1}^{n} \beta_{i} \theta_{i}$ agree on the first $n$ points of $\left(D,<_{D}\right)$ (see $\left.\S 2\right)$. Automatically $g$ and $g_{n}$ agree at zero and one. If $n=2^{k}-1$ and $k \geqslant m$, then $g-g_{n}$ is zero on the set $\left\{j / 2^{k}: 0 \leqslant j \leqslant 2^{k}\right\}$ and linear on each interval $\left(j / 2^{k-1},(j+1) / 2^{k-1}\right)$ with $0 \leqslant j<2^{k-1}$. (The reader may find a graph of $g-g_{n}$ helpful.) Furthermore, $\sup \left|g(t)-g_{n}(t)\right| \leqslant M 2^{-k}$. If $f(t)$ is a function define $f\left(t^{+}\right)=\lim _{s \rightarrow t^{+}} f(s)$ and $f\left(t^{-}\right)=\lim _{s \rightarrow t^{-}} f(s)$. By Lemma 1.1(c), $\left\|g-g_{n}\right\|=\left\|\sum_{i=1}^{n} \alpha_{i} x_{2 i}\right\|$, where the terms of the sequence $\alpha_{1}, \alpha_{2}, \ldots, \alpha_{n}$ are the values $f\left(0^{+}\right), f\left(\left(1 / 2^{k-1}\right)^{-}\right), f\left(\left(1 / 2^{k-1}\right)^{+}\right), f\left(\left(2 / 2^{k-1}\right)^{-}\right), \ldots, f\left(1^{-}\right)$when $f=g-g_{n}$. Since $\left\{x_{2 n}\right\}_{n}$ is orthogonal, $\left\|g-g_{n}\right\| \leqslant 4 M 2^{-k}\left\|\sum_{i=1}^{n} x_{2 i}\right\| \leqslant 4 M \lambda\left(2^{k}\right) / 2^{k} \rightarrow 0$. Hence $g \in X W 2$ and since such functions $g$ are dense in $X F$, we have $X F \subset X W 2$.

Define sequences $\left\{a_{n}\right\},\left\{b_{n}\right\}$ and $\left\{c_{n}\right\}$ by $a_{1}=1 / 4, b_{1}=3 / 4, c_{n}=\left(a_{n}+b_{n}\right) / 2$, $a_{2 n}=a_{n}, b_{2 n+1}=b_{n}, b_{2 n}=\left(a_{n}+c_{n}\right) / 2$ and $a_{2 n+1}=\left(c_{n}+b_{n}\right) / 2$. Then

$$
0<a_{n}=a_{2 n}<c_{2 n}<b_{2 n}<c_{n}<a_{2 n+1}<c_{2 n+1}<b_{2 n+1}=b_{n}<1 \text {. }
$$

Inductively define functions $\left\{\phi_{n}\right\}_{1}^{\infty}$ in $X F$, so that

(1) $\phi_{1}(0)=\phi_{1}(1)=0, \phi_{1}\left(a_{1}\right)=\phi_{1}\left(b_{1}\right)=1$ and $\phi_{1}$ is linear on each of the intervals $\left[0, a_{1}\right],\left[a_{1}, b_{1}\right]$ and $\left[b_{1}, 1\right]$;

(2) $\phi_{2 n}=\phi_{n}$ on $\left[0, b_{2 n}\right], \phi_{2 n}=0$ on $\left[c_{n}, 1\right]$ and $\phi_{2 n}$ is linear on $\left[b_{2 n}, c_{n}\right]$;

(3) $\phi_{2 n+1}=\phi_{2 n}$ on $\left[a_{2 n+1}, 1\right], \phi_{2 n+1}=0$ on $\left[0, c_{n}\right]$ and $\phi_{2 n+1}$ is linear on $\left[c_{n}, a_{2 n+1}\right]$.

(4) For later convenience, define $\phi_{-1} \equiv 1$ and define $\phi_{0}$ so that $\phi_{0}(0)=0, \phi_{0} \equiv 1$ on $\left[a_{1}, 1\right]$ and $\phi_{0}$ is linear on $\left[0, a_{1}\right]$.

Note that $\left\{t: \phi_{n}(t)=1\right\}=\left[a_{n}, b_{n}\right]$, for $n \geqslant 1$, and hence $\left\{\phi_{n}\right\}_{n=1}^{\infty}$ is 1-equivalent to $\left\{\theta_{n}\right\}$ by Lemma 1.1(c). Therefore $X W 2$ is isometric to $\left[\phi_{n}\right]_{n=1}^{\infty} \subset X F$.

Next, we define a projection $P$ on $X F$. The function $P g$ is obtained from $g$ as follows. For $t \in K=\left\{a_{n}\right\} \cup\left\{b_{n}\right\} \cup\left\{c_{n}\right\} \cup\{0,1\},(P g)(t)=g(t)$ and $P g$ is linear on each of the intervals $\left[0, a_{1}\right],\left[b_{1}, 1\right],\left[b_{2 n}, c_{n}\right]$ and $\left[c_{n}, a_{2 n+1}\right]$. Note that $P g$ is defined on $[0,1]$. By Lemma 1.1(c),

$$
\|P g\|=\sup \left\{\left\|\sum_{i=1}^{n} \operatorname{Pg}(j(i)) x_{i}\right\|: j(i) \in K, j(i)<j(i+1), n \geqslant 1\right\}
$$

and since $P g$ and $g$ agree on $K, P$ is a norm one projection. For $n \geqslant-1, P \phi_{n}=\phi_{n}$ so that $\left[\phi_{n}\right]_{n=-1}^{\infty} \subset P(X F)$.

Suppose $g=P g$ and let $h=g-g(0) \phi_{-1}-(g(1)-g(0)) \phi_{0}$. Then $h=P h$ and $h(0)=h(1)=0$. Suppose further that for some $m \geqslant 0, h$ and $g$ are linear on each of the intervals $\left[a_{n}, b_{n}\right]$ with $2^{m} \leqslant n<2^{m+1}$. Inductively choose scalars $\left\{\beta_{n}\right\}$ so that $h$ and $h_{n}=\sum_{i=1}^{n} \beta_{i} \phi_{i}$ agree on the points $\left\{c_{i}\right\}_{i=1}^{n}$. As before, let $M=\sup \left|h^{\prime}(t)\right|$. If $n=2^{k}-1$ with $k>m$, and $c<c^{\prime}$ are adjacent (in the ordering on $I$ ) elements of $\left\{c_{i}\right\}_{i=1}^{n} \cup\{0,1\}$, then $h-h_{n}$ on $\left[c, c^{\prime}\right]$ is at worst a function which is the union of two linear functions. (Again, a graph $h-h_{n}$ may aid the reader.) Furthermore, $\sup \left|h(t)-h_{n}(t)\right| \leqslant M 2^{-k}$ and as before, Lemma 1.1(c) implies that

$$
\left\|h-h_{n}\right\| \leqslant 4 M 2^{-k} \lambda\left(2^{k}\right) \rightarrow 0 \text {. }
$$


Since such functions $g$ are dense in $P(X F)$, we have $\left[\phi_{n}\right]_{n=-1}^{\infty}=P(X F)$. The Projection Principle yields a norm one projection from $\left[\phi_{n}\right]_{n=-1}^{\infty}$ onto $\left[\phi_{-1}, \phi_{0}\right]$ with kernel $\left[\phi_{n}\right]_{n=1}^{\infty}$. Therefore $\left[\phi_{n}\right]_{1}^{\infty}$ is 2-complemented in $X F$ and the proof is complete.

COROllary 3.4. If $J$ is James space, then JW2 contains no subspace isomorphic to $l_{1}$ but does contain a subspace isomorphic to $c_{0}$.

Proof. These statements are true about $J F[11]$.

REMARKs. 1. The author has been told that Corollary 3.4 is in [13].

2. It is easy to check that if $\left\{x_{n}\right\}$ is neighborly and $\left\{x_{2 n}\right\}_{n}$ is equivalent to the usual basis of $l_{1}$, then $\left\{x_{n}\right\}$ is equivalent to the sequence in Example 1.3(b), and thus $\left[x_{n}\right]$ is isomorphic to $l_{1}$. Hence Theorem 3.3 "works" for all but one neighborly basis.

3. The author does not know if $X F \subset X W 2$ is complemented. If true, then Pelczynski's decomposition method implies that the three spaces $X W 2, X F$ and $Y F$ are isomorphic.

Question. For which $X$ are the spaces $X F$ and $X W 2$ isomorphic?

\section{Examples.}

EXAMPLE 4.1. A subsymmetric basis $\left\{e_{n}\right\}$ whose inversion contains a subspace isomorphic to $c_{0}$, but for which $\left[e_{n}\right]$ is reflexive.

Construction. Let $0<\alpha<1$ and $\beta=1-\alpha$. Let the sequences $\left\{a_{n}\right\}$ and $\left\{b_{n}\right\}$ be defined by $a_{n}=n^{-\alpha}$ and $b_{n}=n^{-\beta}$. Let $1<p<\infty$.

The example will be the completion of the space of finitely nonzero scalar sequences with the norm defined by

$$
\left\|\left\{\alpha_{n}\right\}\right\|=\sup \left\{\left(\sum_{i} a_{i}\left|\alpha_{n(i)}\right|^{p}\right)^{1 / p}:\{n(i)\}\right. \text { an increasing sequence }
$$

$$
\text { of positive integers }\} \text {. }
$$

The $\left\{e_{n}\right\}$ will be the usual vector basis. Clearly, $\left\{e_{n}\right\}$ is a subsymmetric basis.

To see $\left[e_{n}\right]$ is reflexive, we will show that this basis is shrinking and boundedly complete. Since $\left\|\sum_{n} \alpha_{n} e_{n}\right\|^{p} \leqslant\left\|\sum_{n=1}^{m} \alpha_{n} e_{n}\right\|^{p}+\left\|\sum_{n=m+1}^{\infty} \alpha_{n} e_{n}\right\|^{p}$, the basis is shrinking. For boundedly completeness, consider the functions

$$
f_{k}\left(\sum_{n} \alpha_{n} e_{n}\right)=\sup \left\{\left(\sum_{i=1}^{k} a_{i}\left|\alpha_{n(i)}\right|^{p}\right)^{1 / p}:\{n(i)\} \text { as before }\right\} .
$$

Now if $\left\{e_{n}\right\}$ is not boundedly complete, there is a block basic sequence $\left\{x_{i}\right\}$ of $\left\{e_{n}\right\}$ so that $\left\|x_{i}\right\|>1$ and $\left\|\sum_{i=1}^{n} x_{i}\right\|<2$ for each $i$ and $n$. There are two cases to consider:

(1) for some $k, \liminf _{i} f_{k}\left(x_{i}\right)>0$, and

(2) for each $k, \liminf _{i} f_{k}\left(x_{i}\right)=0$.

Contradictions are obtained in each case as follows. In case (1), using the unconditionality of $\left\{x_{i}\right\}$ and passing to a subsequence, if necessary, we may assume, for some $k$ and each $i, f_{k}\left(x_{i}\right) \geqslant \varepsilon>0$. Estimate $\left\|\sum_{j=1}^{m} x_{j}\right\|$ by choosing $\{n(i)\}$ so that 
for each $j$ the sequence $\{n(k(j-1)+1), \ldots, n(k j)\}$ is used to obtain $f_{k}\left(x_{j}\right)$. We have the contradiction,

$$
2^{p}>\left\|\sum_{j=1}^{m} x_{j}\right\|^{p} \geqslant \sum_{j=1}^{m} \varepsilon^{p} / j^{\alpha} k^{\alpha} \geqslant \varepsilon^{p} k^{-\alpha} m^{\beta} / \beta \rightarrow \infty .
$$

In case (2), by passing to a subsequence, we may assume the existence of a subsequence $\{k(i)\}$ so that

$$
f_{k(i)}\left(x_{i}\right)=\left\|x_{i}\right\| \text { and }\left\|x_{i+1}\right\|-f_{k(i)}\left(x_{i+1}\right)>1 .
$$

Now choose $\{n(i)\}$ so that the $k(j)+1$ to $k(j+1)$ integers are the $k(j)+1$ to $k(j+1)$ integers in the subsequence which norms $\left\{x_{j}\right\}$. We have the contradiction

$$
2^{p}>\left\|\sum_{j=1}^{m} x_{j}\right\|^{p} \geqslant \sum_{j=1}^{m}\left(\left\|x_{j}\right\|-f_{k(j-1)}\left(x_{j}\right)\right) \geqslant m \rightarrow \infty .
$$

Therefore, $\left[e_{n}\right]$ is reflexive.

To show that $c_{0}$ is in the inversion of $\left\{e_{n}\right\}$, we will need two facts about the sequence $s_{n}=\sum_{i=1}^{n} i^{-\alpha}(n+1-i)^{-\beta}$. Namely that $s_{n}$ converges to a finite limit $s_{\infty}$ and for each $n, s_{n} \leqslant s_{\infty}$. An interesting way to obtain these facts is to use $(n+1)^{\alpha}(n+1)^{\beta} /(n+1)=1$ to rewrite $s_{n}$ as

$$
\sum_{i=1}^{\infty}\left(\frac{i}{n+1}\right)^{-\alpha}\left(1-\frac{i}{n+1}\right)^{-\beta} \frac{1}{n+1}=\int_{0}^{1} \sum_{i=1}^{n} f\left(\frac{i}{n+1}\right) \chi_{A(i)}
$$

where $f(x)=x^{-\alpha}(1-x)^{-\beta}$ and $A(i)=[i /(n+1)-1 / 2(n+1), i /(n+1)+$ $1 / 2(n+1)$ ]. Clearly the functions inside the integral converge pointwise to $f$. Using $f^{\prime \prime}(x)>0$, one obtains $s_{n} \leqslant \int_{0}^{1} f$ and Fatou's lemma implies $\int_{0}^{1} f \leqslant \lim \inf s_{n}$. Hence $\lim s_{n}=\int_{0}^{1} f=s_{\infty}$ and we also have $\int_{0}^{1} f<\infty$.

We will write the inversion of $\left\{e_{n}\right\}_{n=1}^{\infty}$ as $\left\{e_{n}\right\}_{n=-\infty}^{-1}$. For $-\infty<n \leqslant-1$, let $\alpha_{n}=(-n)^{-\beta / p}$. A subspace isomorphic to $c_{0}$ is obtained by finding a decreasing sequence of negative integers $\{m(i)\}_{i=1}^{\infty}$, and letting $m(0)=0$, so that the block basic sequence $x_{n}=\sum_{j=m(n)}^{m(n-1)-1} \alpha_{j} e_{j}$ satisfies $\left\|x_{n}\right\|>s_{\infty}-\varepsilon$ and $\left\|\sum_{i=1}^{n} x_{i}\right\| \leqslant s_{\infty}$, for each $n$. The second inequality is automatic. To satisfy the first inequality, inductively pick $m(i+1)<m(i)$ so that $s_{-m(i+1)}>s_{\infty}-\varepsilon / 2$ and

$$
(m(i)-m(i+1))^{-\alpha} \sum_{j=m(i)}^{-1} \alpha_{j}^{p}<\varepsilon / 2 .
$$

This completes the construction.

REMARKS. 1. If $\left\{x_{n}\right\}$ is the inversion of the basis $\left\{e_{n}\right\}$ in the example, then $\left[x_{n}\right]^{\prime}$ is separable. If this were not true, $\left\{x_{n}\right\}$ would contain a block basic sequence equivalent to the usual $l_{1}$-basis, which it cannot because of the upper $l_{p}$-estimate used to show that $\left\{e_{n}\right\}$ is shrinking.

2. If $\left\{f_{n}\right\}$ are the coefficient functionals to the $\left\{e_{n}\right\}$ constructed above, then $\left\{f_{n}\right\}$ is a subsymmetric basis with $\left[f_{n}\right]$ reflexive, but whose inversion contains a complemented subspace which is isomorphic to $l_{1}$.

3. Example 4.1 is another example of a subsymmetric basis which is not symmetric (see [12, p. 115]). 
4. It may be of some interest to note that the Lorentz sequence space $d\left(\left\{a_{n}\right\}, p\right)$ with $\left\{a_{n}\right\}$ and $p$ as in the example, is uniformly convex [1].

EXAMPLE 4.2. A neighborly basis that spans a quasireflexive space and whose inversion spans a space which is not quasireflexive.

Construction. Let $\left\{u_{n}\right\}$ be the subsymmetric basis contructed in Example 4.1. Let $\left\{e_{n}\right\}$ be the neighborly basis with norm $\rho(\cdot)$ constructed from $\left\{e_{2 n}\right\}=\left\{u_{n}\right\}$ as in Proposition 1.4 or equivalently the space $J\left(\left[u_{n}\right]\right)$ of Example $1.3(\mathrm{~g})$. The inversion of $\left\{e_{n}\right\}$ contains the inversion of $\left\{u_{n}\right\}$ and hence a subspace isomorphic to $c_{0}$, and is thus not quasireflexive.

The basis $\left\{e_{n}\right\}$ has an upper $l_{p}$-estimate like the one used to show $\left\{u_{n}\right\}$ is shrinking. Thus $\left\{e_{n}\right\}$ is shrinking. The proof that $\left[e_{n}\right]$ is quasireflexive, that is, it does not have a skipped block basic sequence equivalent to the usual $l_{1}$-basis, is similar to the proof that $\left\{u_{n}\right\}$ is boundedly complete and is omitted.

REMARKS. 1. If $\left\{x_{n}\right\}$ is the inversion of $\left\{e_{n}\right\}$, then the "upper $l_{p}$-estimate" again shows $\left[x_{n}\right]^{\prime}$ is separable. Hence if $E=\left[e_{n}\right]$, then $E^{\omega+1}$ is separable, but $E^{\omega+2}$ is nonseparable. This is one of the examples promised after Corollary 2.7.

2. If $\left\{f_{n}\right\}$ are the coefficient functionals to the $\left\{e_{n}\right\}$, then $\left\{f_{n}\right\}$ is an ESA basis of a quasireflexive space, whose inversion contains $l_{1}$. Thus if $F=\left[f_{n}\right], F^{\omega}$ is separable but $F^{\omega+1}$ is nonseparable. This is the second of the examples promised in the remarks after Corollary 2.7.

The rest of the examples use the following theorem.

THEOREM 4.3. Let $\left(\left\{e_{n}\right\},\|\cdot\|_{1}\right)$ be a neighborly basis and norm so that $\left\|e_{n}\right\|_{1}=1$ and, for some constant $C$, some $p$ with $1<p<\infty$, and for any increasing sequence of integers $\{m(i)\}$ with $m(0)=0$ and scalars $\left\{\alpha_{n}\right\}$

$$
\left\|\sum \alpha_{n} e_{n}\right\|_{1}^{p} \leqslant C^{p} \sum_{i}\left\|\sum_{n=m(i-1)+1}^{m(i)} \alpha_{n} e_{n}\right\|_{1}^{p} .
$$

Then there is a quasireflexive Banach space $X$ of order one with a basis, so that $X^{\omega}$ is isomorphic to $X \oplus Y$, where $Y$ is the closed linear span of the inversion of $\left\{e_{n}\right\}$.

Before proving Theorem 4.3, we list some corollaries.

COROLlaRY 4.4. There is a quasireflexive Banach space $X$ so that $X^{2 \omega+1}$ is separable but $X^{2 \omega+2}$ is nonseparable and so that $\left[X^{\prime}\right]^{2 \omega}$ is separable but $\left[X^{\prime}\right]^{2 \omega+1}$ is nonseparable.

Proof. Let $\left\{e_{n}\right\}$ be the inversion of the basis constructed in Example 4.2 (which we will call $\left.\left\{x_{n}\right\}\right)$ and let $\|\cdot\|_{1}$ be the neighborly norm given by this inversion. Let $X$ be the space given by the theorem and let $Y=\left[x_{n}\right]$. It follows that $X^{\omega} \approx X \oplus Y$ and $\left[X^{\prime}\right]^{\omega} \approx X^{\prime} \oplus Y^{\prime}$. Hence $X^{2 \omega+1} \approx X^{\omega+1} \oplus Y^{\omega+1} \approx[X \oplus Y]^{\prime} \oplus Y^{\omega+1}$ is separable, but $X^{2 \omega+2}$ contains $Y^{\omega+2}$ and is nonseparable. Similarly $\left[X^{\prime}\right]^{2 \omega}$ is separable but $\left[X^{\prime}\right]^{2 \omega+1}$ is nonseparable. These are the last of the examples promised after Corollary 2.7.

COROLlaRY 4.5. There is a quasireflexive space $X$ with $X^{\omega} \approx X \oplus c_{0}$ and $\left[X^{\prime}\right]^{\omega} \approx$ $X^{\prime} \oplus l_{1}$. 
Proof. Take $\left\{e_{n}\right\}$ to be the usual basis for $c_{0}$ and take $\|\cdot\|_{1}$ to be the usual sup norm. Apply the theorem to obtain $X$.

EXAMPLE 4.6. There is a quasireflexive Banach space $X$ so that no basic sequence in $X$ is isomorphic to either a neighborly or ESA sequence. Furthermore, no basis of a quotient of $X$ is isomorphic to either a neighborly or ESA sequence.

Proof. $X$ is the space of Corollary 4.5. Since $\left\{f_{n}\right\}$ ESA implies that $\left\{f_{n}-f_{n+1}\right\}$ is neighborly, it suffices to show that neither $X$ nor $X^{\prime}$ have basic sequences isomorphic to a neighborly sequence.

Suppose $\left\{e_{n}\right\}$ is a neighborly sequence, $E=\left[e_{n}\right]$ and $T: E \rightarrow X$ is isomorphic to an inclusion map. Then $T^{(\omega)}: E^{\omega} \rightarrow X^{\omega}$ is also an isomorphism into.

We must assume that $X^{\omega}$ has a representation similar to the one constructed for $E^{\omega}$ in $\S 2$. That this is true is contained in the proof of Theorem 4.3. Let $\left\{x_{n}\right\}$ be the basis for $X$ constructed by the theorem. Then $X^{\omega}$ can be considered as the closed linear span of $\left(\left\{x_{n}\right\}_{n=1}^{\infty} \cup\left\{x_{n}\right\}_{n=-\infty}^{-1} \cup\left\{\sum_{1}^{\infty} x_{n}+\Sigma_{-\infty}^{-1} x_{n}\right\}\right)$ and similarly for $E^{\omega}$. The map $T^{(2)}: E^{2} \rightarrow X^{2}$ must send $\Sigma_{1}^{\infty} e_{n}+\Sigma_{-\infty}^{-1} e_{n}$ to something not in $X$, that is, to something of the form $\Sigma_{1}^{m} d_{n} x_{n}+\beta\left(\Sigma_{1}^{\infty} x_{n}+\Sigma_{-\infty}^{-1} x_{n}\right)$ with $\beta \neq 0$.

If $J_{E}: E \rightarrow E^{2}, J_{X}: X \rightarrow X^{2}$ are the canonical injections, we have $T^{(4)} J_{E}^{(2)}=J_{X}^{(2)} T^{(2)}$. Hence $T^{\omega}$ maps $\Sigma_{1}^{\infty} e_{n}+\Sigma_{-\infty}^{-2} e_{n}$ to $\sum_{1}^{m} \alpha_{n} x_{n}+\beta\left(\sum_{1}^{\infty} x_{n}+\Sigma_{-\infty}^{-2} x_{n}\right)$. Thus $T^{\omega} e_{-1}=x_{-1}$. Continuing by induction, $T^{(\omega)} e_{n}=x_{n}$, for $n \leqslant-1$. Hence the inversion of $\left\{e_{n}\right\}$ is equivalent to $\left\{x_{n}\right\}_{n=-\infty}^{-1}$. But inspection of the proof of Theorem 4.3 for this $X$ shows $\left\{x_{n}\right\}_{n=-\infty}^{-1}$ is equivalent to the usual basis for $c_{0}$. Thus $\left\{e_{n}\right\}_{1}^{\infty}$, its inversion, is equivalent to the usual $c_{0}$-basis. But this is impossible since $\left[e_{n}\right]$ is a subspace of $X$ and $X$ is quasireflexive. The proof for $X^{\prime}$ is done similarly.

Proof of Theorem 4.3. Let $\left(\left\{e_{n}\right\},\|\cdot\|_{1}\right)$ satisfying the hypothesis be given. The space $X$ will be the completion of a new norm $\|\cdot\|$ on the linear span of $\left\{e_{n}\right\}$. Some preliminary definitions are needed to define $\|\cdot\|$.

A sequence of projections $\left\{P_{i}\right\}$ is said to be admissible if there is an increasing sequence of integers $\{n(i)\}_{i=0}^{\infty}$, with $n(0)=0$, so that

$$
P_{i}\left(\sum \alpha_{j} e_{j}\right)=\sum_{j=n(i-1)+1}^{n(i)} \alpha_{j} e_{j} .
$$

Consider $\|x\|_{2}=\inf \left\{\left(\sum_{i}\left\|P_{i} x\right\| P\right)^{1 / p}:\left\{P_{i}\right\}\right.$ admissible $\}$. The upper estimate on $\|\cdot\|_{1}$ implies that $\|x\|_{1} \geqslant\|x\|_{2} \geqslant C^{-1}\|x\|_{1}$. Hence $\|\cdot\|_{2}$ is equivalent to $\|\cdot\|_{1}$. Furthermore, $\left\{e_{n}\right\}$ is neighborly in $\|\cdot\|_{2}$ and if $\left\{P_{i}\right\}$ is admissible $\|x\|_{2}^{p} \leqslant \Sigma_{i}\left\|P_{i} x\right\| \frac{p}{2}$. Thus we may and do assume $\|\cdot\|_{1}=\|\cdot\|_{2}$, that is, the constant $C$ in the upper estimate on $\|\cdot\|_{1}$ is 1 .

A finite increasing sequence of integers $\{k(i)\}_{i=0}^{m}$ is said to be reasonable if $k(0)=0$ and $k(1) \geqslant m$. An element $x$ of the linear span $\left\{e_{n}\right\}$ is acceptable if there are scalars $\left\{\alpha_{i}\right\}_{i=1}^{m}$, integers $0<r<s$, and a reasonable $\{k(i)\}_{i=0}^{m}$ so that $P\left(\Sigma \beta_{n} e_{n}\right)$ $=\sum_{n=r}^{s} \beta_{n} e_{n}$ and

$$
x=P\left(\sum_{i=1}^{m} \alpha_{i}\left(\sum_{j=k(i-1)+1}^{k(i)} e_{j}\right)\right)
$$


Let the function $f$ be the restriction of the norm $\|\cdot\|_{1}$ to the acceptable elements. For convenience, we define $f(x)=\infty$ if $x$ is not acceptable.

Define a function $g$ on the linear span $\left\{e_{n}\right\}$ by

$$
g(x)=\inf \left\{\left(\sum_{i}\left(f\left(P_{i} x\right)\right)^{p}\right)^{1 / p}:\left\{P_{i}\right\} \text { admissible }\right\} .
$$

Note that $g(x)$ is real-valued positive homogeneous and positive definite on span $\left\{e_{n}\right\}$. Also $g(x)=\|x\|_{1}$ if $x$ is acceptable. In general $g$ is not a norm. Finally, we define

$$
\|x\|=\inf \left\{\sum_{i=1}^{n} g\left(x_{i}\right): x=\sum_{i=1}^{n} x_{i}\right\} .
$$

The function $\|\cdot\|$ is a norm on the linear span of $\left\{e_{n}\right\}$ and the required $X$ is the completion of this space.

Showing $X$ has the desired properties requires some preliminary observations. If $x$ is in the linear span $\left\{e_{n}\right\}$ and if $\left\{P_{i}\right\}$ is admissible, then $x$ acceptable implies $P_{i} x$ is acceptable. Thus if $h$ is one of $f, g$ or $\|\cdot\|$, we have $h\left(P_{i} x\right) \leqslant h(x)$, and $\left\{e_{n}\right\}$ is a bimonotone basis for $X$. Furthermore, this implies that both infs in the definition of $g$ and $\|\cdot\|$ are attained, that is the inf is a minimum on elements in the linear span $\left\{e_{n}\right\}$.

Next, we show that if $x$ is the linear span $\left\{e_{n}\right\},\left\{P_{i}\right\}$ is admissible, $\left\|P_{1} x\right\|=\left\|P_{2} x\right\|$ $=\cdots=\left\|P_{n} x\right\|$, and $0=\left\|P_{n+1} x\right\|=\left\|P_{n+2} x\right\|=\ldots$, then

$$
\|x\|^{p} \leqslant \sum_{i}\left\|P_{i} x\right\|^{p}
$$

Certainly $g(x)^{p} \leqslant \Sigma_{i} g\left(P_{i} x\right)^{p}$ for any $x$ in $X$. To see (*) is true, pick $y_{j}^{i}$ and $m(i)$ so that $P_{i}\left(y_{j}^{i}\right)=y_{j}^{i}, P_{i} x=\sum_{j=1}^{m(i)} y_{j}^{i}$ and $\left\|P_{i} x\right\|=\sum_{j=1}^{m(i)} g\left(y_{j}^{i}\right)$. Since $g$ is positive homogeneous, we can replace the $\left\{y_{j}^{i}\right\}$ and $\{m(j)\}$ with $\left\{z_{j}^{i}\right\}$ and $m$ so that $P_{i}\left(z_{j}^{i}\right)=z_{j}^{i}$, $P_{i} x=\sum_{j=1}^{m} z_{j}^{i},\left\|P_{i} x\right\|=\sum_{j=1}^{m} g\left(z_{j}^{i}\right), g\left(z_{j}^{i}\right)=g\left(z_{j}^{k}\right)$ for $1 \leqslant i, k \leqslant n$ and each $z_{j}^{i}$ is some positive scalar multiple of some $y_{k}^{i}, 1 \leqslant k \leqslant m(i)$. Now

$$
\begin{aligned}
\|x\| & \leqslant \sum_{j=1}^{m} g\left(\sum_{i=1}^{n} z_{j}^{i}\right) \leqslant \sum_{j=1}^{m} n^{1 / p} g\left(z_{j}^{1}\right) \\
& =n^{1 / p}\left\|P_{1} x\right\|=\left(\sum_{i=1}^{n}\left\|P_{i} x\right\|^{p}\right)^{1 / p},
\end{aligned}
$$

and $(*)$ is proved. It is straightforward to show that $(*)$ implies that the basis $\left\{e_{n}\right\}$ is shrinking, and it was to this purpose the function $g$ was introduced.

Again, let $x$ be in the linear span of $\left\{e_{n}\right\}$. It follows from $\|x\|_{1} \leqslant f(x)$ and the given estimate on $\|\cdot\|_{1}$ that $\|x\|_{1} \leqslant g(x)$ and hence $\|x\|_{1} \leqslant\|x\|$. Conversely, if $x$ is acceptable, $\|x\| \leqslant g(x)=f(x)=\|x\|_{1}$, and hence $\|\cdot\|$ and $\|\cdot\|_{1}$ are equal on the acceptable elements. Now, $1=\left\|e_{1}\right\| \leqslant\left\|\sum_{i=1}^{n} e_{i}\right\| \leqslant\left\|\sum_{i=1}^{n} e_{i}\right\|_{1}=\left\|e_{1}\right\|_{1}=1$, since $\sum_{i=1}^{n} e_{i}$ is acceptable. Therefore, the formal sum $\Sigma e_{i}$ belongs to the bidual of $X$, and $X$ is not reflexive. Showing that $X^{2}$ is the span of $X \cup\left\{\sum e_{i}\right\}$ is the most delicate part of the proof, and will be done last. 
So assume

(\#) $X$ is quasireflexive of order one.

We will describe the space $X^{\omega}$. The basic idea is to show that the description in $\$ 2$ works for $X^{\omega}$ as well. Indeed, although the basis $\left\{e_{n}\right\}$ with $\|\cdot\|$ is not neighborly, it has half of the required property. Having spent time in the South, the author defines a basis $\left\{e_{n}\right\}$, in the norm $\|\cdot\|$, to be right neighborly if for each finitely nonzero sequence of scalars $\left\{\alpha_{n}\right\}$ and integer $i \geqslant 0$ (again $\alpha_{0}=0$ )

$$
\left\|\sum \alpha_{n} e_{n}\right\| \geqslant\left\|\sum_{n=1}^{i} \alpha_{n} e_{n}+\alpha_{i} e_{i+1}+\sum_{n=i+2}^{\infty} \alpha_{n} e_{n}\right\| \text {. }
$$

That is, the norm of a sequence is not increased if some scalar replaces the scalar which is its neighbor to the right. The basis $\left\{e_{n}\right\}$ is right neighborly in $\|\cdot\|$, since $\sum \alpha_{n} e_{n}$ acceptable implies $\sum_{n=1}^{i} \alpha_{n} e_{n}+\alpha_{i} e_{i+1}+\sum_{n=i+2}^{\infty} \alpha_{n} e_{n}$ is acceptable, and $\|\cdot\|_{1}$ is neighborly.

Let $L$ be the set of integers with the unusual ordering $n \leqslant_{L} m$ if $n \leqslant m \leqslant 0$ or if $0<n \leqslant m$ or if $n>0 \geqslant m$. For $n \in L$, let $e_{n}$ be the function that is one at $n$ and zero otherwise. Let $\theta$ be the function which is constantly one on $L$. Let $Y$ be the linear span of $\{\theta\} \cup\left\{e_{n}\right\}_{n \in L}$. As before we extend the neighborly norm $\|\cdot\|_{1}$ to $Y$. The norm $\|\cdot\|$ is extended to $Y$ as follows: $\left\|\sum_{i=1}^{n} \alpha_{i} e_{i}+\beta \theta+\sum_{i=-m}^{0} \alpha_{i} e_{i}\right\|$ is defined for finite positive integers $m, n$ to be the limit as $j \rightarrow \infty$ of

$$
s_{j}=\left\|\sum_{i=1}^{n} \alpha_{i} e_{i}+\beta \sum_{i=1}^{j} e_{i}+\sum_{i=-m}^{0} \alpha_{i} e_{j+i}\right\| \text {. }
$$

Right neighborly implies that the $s_{j}$ are eventually decreasing and thus the limit exists. Also note that as $j$ increases, $\beta \sum_{i=1}^{j} e_{i}+\sum_{i=-m}^{0} \alpha_{i} e_{j+i}$ eventually becomes and remains acceptable. Hence on the span of $\{\theta\} \cup\left\{e_{n}\right\}_{n=-\infty}^{0}$ the norms $\|\cdot\|_{1}$ and $\|\cdot\|$ are equal. In particular, the $\|\cdot\|$-completion of $\operatorname{span}\left\{e_{n}\right\}_{n=-\infty}^{0}$ is isometric to the inversion of $\left\{e_{n}\right\}_{n=1}^{\infty}$ in $\|\cdot\|_{1}$.

Let $\left\{\theta_{n}\right\}_{n=0}^{\infty} \subset Y$ be defined by $\theta_{0}=\theta$ and $\theta_{n+1}=\theta_{n}-e_{-n}$. Since $\|\cdot\|$ and $\|\cdot\|_{1}$ agree on $\operatorname{span}\left\{\theta_{n}\right\}$ and $\left\{\theta_{n}\right\}$ is a monotone basic sequence in $\|\cdot\|_{1}$, we have

(1) $\left\{\theta_{n}\right\}$ is a monotone basic sequence in $\|\cdot\|$.

Let $m, n$ be given and consider $\sum_{i=0}^{m} \beta_{i}\left(\sum_{j=1}^{k-i} e_{j}\right)$ and $\sum_{i=0}^{m} \beta_{i}\left(\sum_{j=n+1}^{k-i} e_{j}\right)$. For large $k$, both these vectors are acceptable and since they have the same $\|\cdot\|_{1}$-value their $\|\cdot\|$-values are equal too. Since $\left\{e_{n}\right\}$ is bimonotone in $\|\cdot\|$, for large $k$,

$$
\left\|\sum_{i=1}^{n} \alpha_{i} e_{i}+\sum_{i=0}^{m} \beta_{i}\left(\sum_{j=1}^{k-i} e_{j}\right)\right\| \geqslant\left\|\sum_{i=0}^{m} \beta_{i}\left(\sum_{j=n+1}^{k-i} e_{j}\right)\right\|=\left\|\sum_{i=0}^{m} \beta_{i}\left(\sum_{j=n+1}^{k-i} e_{j}\right)\right\| .
$$

Therefore

(2) the projection $\sum_{i=1}^{n} \alpha_{i} e_{i}+\sum_{i=0}^{m} \beta_{i} \theta_{i} \rightarrow \sum_{i=0}^{m} \beta_{i} \theta_{i}$ has norm one in $(Y,\|\cdot\|)$.

We complete the proof (modulo (\#)) by showing $X^{2}$ (resp. $X^{2 n+2}, n \geqslant 1$ ) is isometric with $Y_{2}$ (resp., $\left.Y_{2 n+2}\right)=$ the completion of the span of $\{\theta\} \cup\left\{e_{i}\right\}_{i=1}^{\infty}$ (resp., $\left.\{\theta\} \cup\left\{e_{i}\right\}_{i=-n+1}^{\infty}\right)$ in $(Y,\|\cdot\|)$ and that the inclusion $Y_{2 n} \subset Y_{2 n+2}$ is the canonical injection $X^{2 n} \rightarrow X^{2 n+2}$. This can be done as in $\S 2$, since (1) and (2) are true. 
Finally it remains to show (\#). Choose $\varepsilon>0$ so that $\delta=\left[(1+\varepsilon)^{p}-1\right]^{1 / p}<1 / 6$. If (\#) is false, then as in the proof of Proposition 1.2(d), there is a scalar sequence $\left\{\alpha_{n}\right\}$ and an increasing sequence of integers $\{n(i)\}$ with $n(0)=0$, so that $\alpha_{n(i)}=0$ and if $b_{i}=\sum_{j=n(i-1)+1}^{n(i)} \alpha_{j} e_{j}$, then $\left\|b_{i}\right\|>1$ and $\left\|\sum_{i=r}^{s} b_{i}\right\|<1+\varepsilon$, for each $i$, and $r<s$ positive integers.

We will obtain a contradiction by showing that if $m$ is large enough (its precise value will be given later) there is an integer $1 \leqslant j \leqslant m-2$ and a constant $c$, so that if $w=\sum_{i=n(j)+1}^{n(j+2)-1} c e_{i}$ then $\left\|\sum_{i=j+1}^{j+2} b_{i}-w\right\|<3 \delta$ and hence $\|w\|>1-3 \delta$. Let $\|\cdot\|_{\infty}$ be the usual sup norm. Since $\|\cdot\|_{1}$ is a normalized neighborly norm, $\|w\|=\|w\|_{1}$ $=\|w\|_{\infty}=|c|>1-3 \delta$. Since $\left\{e_{n}\right\}$ is bimonotone in $\|\cdot\|$,

$$
\left|\alpha_{n(j+1)}-c\right| \leqslant\left\|\sum_{i=j}^{j+2} b_{i}-w\right\|_{\infty} \leqslant\left\|\sum_{i=j}^{j+2} b_{i}-w\right\|<3 \delta .
$$

Hence $\left|\alpha_{n(j+1)}\right| \geqslant|c|-\left|\alpha_{n(j+1)}-c\right|>1-6 \delta>0=\alpha_{n(j+1)}$, a contradiction.

For this large $m$, let $\left\{x_{j}\right\}_{j=1}^{n}$ be so that $\sum_{i=1}^{m} b_{i}=\sum_{j=1}^{n} x_{j}$ and $\left\|\sum_{i=1}^{m} b_{i}\right\|=$ $\sum_{j=1}^{n} g\left(x_{j}\right)$. For each $1 \leqslant j \leqslant n$, let $\left\{P_{s}^{j}\right\}_{s}$ be an admissible sequence of projections so that $g\left(x_{j}\right)=\left(\Sigma_{x} f\left(P_{s}^{j} x_{j}\right)^{p}\right)^{1 / p}$ and let $y_{s}^{j}=P_{s}^{j} x_{j}$. Clearly each $y_{s}^{i}$ is acceptable.

Let $Q$ (resp. $R$ ) be the projection which sends $\sum_{i} \beta_{i} e_{i}$ to $\sum_{i=1}^{n(1)} \beta_{i} e_{i}$ (resp. $\left.\sum_{i=n(m-1)+1}^{\infty} \beta_{i} e_{i}\right)$. Let $A$ (resp. $C$ ) be the set of $(j, s)$ so that $Q y_{s}^{j}=0$ (resp. $R y_{s}^{j}=0$ ) and $1 \leqslant j \leqslant n$. Let $B$ be the set of $(j, s)$ with $1 \leqslant j \leqslant n$ and $(j, s) \notin A \cup C$. Observe that if $(j, s) \in B$, then the support of $y_{s}^{j}$ includes both integers less than $n(1)+1$ and integers greater than $n(m-1)$. Thus for each $j$, there is at most one $s$ with $(j, s) \in B$. For $1 \leqslant j \leqslant n$, define $a_{j}$ (resp. $\left.c_{j}, \xi_{j}, \eta_{j}\right)$ to be $\left(\sum_{(j, s) \in D} f\left(y_{s}^{j}\right)^{p}\right)^{1 / p}$ when $D=A$ (resp. $C, A^{c}, C^{c}$ ). (Here $A^{c}$ denotes the complement of the set $A$ in the set $\{(j, s): 1 \leqslant j \leqslant n, s \geqslant 1\}$.)

The estimate for $\left\|\Sigma_{1}^{m} b_{i}\right\|$ can be rewritten as

$$
1<\sum_{j}\left(a_{j}^{p}+\xi_{j}^{p}\right)^{1 / p}=\sum_{J}\left(c_{j}^{p}+\eta_{j}^{p}\right)^{1 / p}<1+\varepsilon .
$$

Since $b_{1}=\Sigma_{j} Q x_{j}$ and $b_{m}=\Sigma_{j} R x_{j}, 1 \leqslant\left\|b_{1}\right\| \leqslant \Sigma_{j} g\left(Q x_{j}\right) \leqslant \Sigma_{j}\left(\Sigma_{s} f\left(Q y_{s}^{j}\right)^{p}\right)^{1 / p} \leqslant$ $\Sigma_{j}\left(\Sigma_{(j, s) \notin A} f\left(y_{s}^{j}\right)^{p}\right)^{1 / p}=\Sigma_{j} \xi_{j}$, and similarly $1<\Sigma_{j} n_{j}$. The triangle inequality for the $l_{p}$-norm in a two-dimensional space implies that

$$
\left(\left(\sum_{j} a_{j}\right)^{p}+\left(\sum_{j} \xi_{j}\right)^{p}\right)^{1 / p} \leqslant \sum_{j}\left(a_{j}^{p}+\xi_{j}^{p}\right)^{1 / p}<1+\varepsilon .
$$

Hence both $\Sigma_{j} a_{j}$ and $\Sigma_{j} c_{j}$ are less than $\left[(1+\varepsilon)^{p}-1\right]^{1 / p}=\delta$.

Let $z=\Sigma_{j} \Sigma_{(j, s) \in B} y_{s}^{j}$. For integers $1 \leqslant q \leqslant r \leqslant m$ let $V_{q}^{r}$ be the projection which sends $\sum \beta_{i} e_{i}$ to $\sum_{i=n(q-1)+1}^{n(r)-1} \beta_{i} e_{i}$. Note that $V_{q}^{r}\left(\sum_{i=1}^{m} b_{i}\right)=\sum_{i=q}^{r} b_{i}$. Consider $\Omega=$ $\sum_{i=q}^{r} b_{i}-V_{q}^{r} z$. Since $\Omega=\Sigma_{j}\left(\Sigma_{(j, s) \notin B} V_{q}^{r} y_{s}^{j}\right)$ and the $l_{1}$-norm dominates the $l_{p}$-norm, we have

$$
\|\Omega\| \leqslant \sum_{j}\left(\sum_{(j, x) \notin A \cup C} f\left(y_{s}^{j}\right)^{p}\right)^{1 / p} \leqslant \sum_{j}\left(a_{j}^{p}+c_{j}^{p}\right)^{1 / p} \leqslant \sum_{j}\left(a_{j}+c_{j}\right)<2 \delta .
$$


From the collection $\left\{y_{s}^{j}:(j, s) \in B\right\}$ we obtain $\left\{z_{\nu}: \nu \in T\right\}$ so that $z=\Sigma_{\nu \in T} z_{\nu}$, for each $y_{s}^{j}$ there is an integer $i \geqslant 0$ such that for $2^{i}$ elements of $T, 2^{i} z_{\nu}=y_{s}^{j}$, and $\max _{\nu \in T}\left\|z_{\nu}\right\|_{1} \leqslant 2 \min _{\nu \in T}\left\|z_{\nu}\right\|_{1}$. We have

$$
1+\varepsilon>\left\|\sum_{1}^{m} b_{i}\right\|=\sum_{j}\left(\sum_{s} f\left(y_{s}^{j}\right)^{p}\right)^{1 / p} \geqslant \sum_{j} \sum_{(j, s) \in B} f\left(y_{s}^{j}\right)=\sum_{\nu \in T}\left\|z_{\nu}\right\|_{1} .
$$

Hence if $T$ has $p$ elements for $\nu \in T$, then

$$
p\left\|z_{\nu}\right\|_{1}<p \max _{\nu \in T}\left\|z_{\nu}\right\|_{1} \leqslant 2 \sum_{\nu \in T}\left\|z_{\nu}\right\|_{1}<2(1+\varepsilon) .
$$

Since $z_{\nu}$ is acceptable there is a reasonable sequence $\{k(j, \nu)\}_{j=0}^{l(\nu)}$, integers $1 \leqslant \xi<\eta$ and scalars $c(j, \nu)$ so that

$$
z_{\nu}=P_{\xi}^{n}\left(\sum_{j=1}^{l(\nu)} c(j, \nu) \sum_{i=k(j-1, \nu)+1}^{k(j, \nu)} e_{i}\right) .
$$

Since each $z_{\nu}$ is a multiple of $y_{s}^{j}$ with $(j, s) \in B, \xi \leqslant n(1)$ and $\eta \geqslant n(m-1)$.

Let $0<\lambda<1$, so that $2(1+\varepsilon)(1-\lambda)<\delta$, let $\mu=3$ and let $m$ be the integer given by Lemma 4.7 (below) for this $\{n(i)\}, \lambda$ and $\mu$. Then there is a subset $S$ of $T$ with at least $\lambda p$-elements and an integer $j$ with $1 \leqslant j \leqslant m-3$ so that it is false that $n(j)<k(i, \nu) \leqslant n(j+3)$ for $\nu \in S$ and $1 \leqslant i \leqslant l(\nu)$.

It follows that

$$
w=V_{j+1}^{j+2}\left(\sum_{\nu \in s} z_{\nu}\right)=\sum_{i=n(j)+1}^{n(j+2)} c e_{i}
$$

for some constant $c$. Also, we have

$$
\begin{aligned}
\left\|\sum_{i=j+1}^{j+2} b_{i}-w\right\| & \leqslant\left\|\sum_{i=j+1}^{j+2} b_{i}-V_{j+1}^{j+2} z\right\|+\left\|\sum_{\nu \notin S} z_{\nu}\right\| \leqslant 2 \delta+\sum_{\nu \notin S}\left\|z_{\nu}\right\|_{1} \\
& \leqslant 2 \delta+(1-\lambda) p\left\|z_{\nu}\right\|_{1} \leqslant 2 \delta+(1-\lambda) 2(1+\varepsilon)<3 \delta .
\end{aligned}
$$

This was shown to lead to a contradiction. Thus to complete the proof of Theorem 4.3 it suffices to prove the following lemma.

LEMMA 4.7. For each increasing sequence of integers $\{n(i)\}_{i=0}^{\infty}$ with $n(0)=0$, each $0 \leqslant \lambda<1$ and positive integer $\mu$ there is a positive integer $m$, so that for each integer $p$ and each p-element list $T$ of reasonable sequences, there is an integer $j$ with $0 \leqslant j \leqslant m$ $-\mu$ and $a$ sublist $S$ of $T$ with at least $\lambda$ p-elements so that if the integer $k$ belongs to $a$ reasonable sequence in $S$, then it is false that $n(j)<k \leqslant n(j+\mu)$.

Proof. It suffices to prove the lemma with $\mu=1$, since we can replace $\{n(i)\}_{i=0}^{\infty}$ with $\{n(\mu i)\}_{i=0}^{\infty}$. Also it suffices to prove the lemma with $\lambda=1 / 2$. Indeed, then we can prove, by induction on $k$, that the lemma is true for $\lambda \leqslant 1 / 2+1 / 2^{2}$ $+\cdots+1 / 2^{k}$ as follows. Let $\{n(i)\}_{i=0}^{\infty}$ be given, let $m(0)=0$ and inductively choose $m(i+1)$ so that it is the $m$ given by the lemma for $\mu=1$ and $\lambda=1 / 2$ and sequence $\{n(j): j=m(\nu)$ for $0 \leqslant \nu \leqslant i$ or $j=m(i)+\nu$ for $\nu \geqslant 1\}$. Apply the inductive hypothesis to the sequence $\{n(m(i))\}_{i=0}^{\infty}$. If $T$ is a $p$ element list of reasonable 
sequences, then for some $j$, at most $p / 2^{k}$ of these reasonable sequences have an integer between $n(m(j))$ and $n(m(j+1))$. And for some $0 \leqslant i<m(j+1)-m(j)$ at most half of those "bad" reasonable sequences have an integer between $n(m(j)+i)$ and $n(m(j)+i+1)$.

Thus assume $\lambda=1 / 2$ and $\mu=1$. Let $\{n(i)\}_{i=0}^{\infty}$ be given. The claim is that $m=2(n(1))+2$ works. If this is not true there are counterexamples and of all these counterexamples there is a $p$ element list $T$ so that $p$ is minimal. The method of proof will be to reduce $T$ to another counterexample of a special form.

First observe that the deletion of any nonzero integer from the reasonable sequence $\{k(j)\}_{j=0}^{r}$, with $r \geqslant 2$, produces another reasonable sequence. The deletion of $k(1)$ from $\{k(j)\}_{j=0}^{1}$ is the same as throwing the sequence away. This second case will not happen anytime we use deletion below since it would produce a counterexample with fewer than $p$ elements. Using deletion as necessary, we may assume that each reasonable sequence $\{k(j)\}_{j=0}^{r}$ in $T$ has at most one $j \geqslant 1$ with $n(i-1)<k(j)$ $\leqslant n(i)$ for each $i \geqslant 1$. Let $q=[p / 2]+1$. Since $T$ is a counterexample, for each $1 \leqslant i \leqslant m$, there are at least $q$ elements of $T$ containing an integer $k$ with $n(i-1)<$ $k \leqslant n(i)$. Again using deletion we may assume that for each $1 \leqslant i \leqslant m$, there are exactly $q$ elements of $T$ containing an integer $k$ with $n(i-1)<k \leqslant n(i)$. Also we may assume that no element of $T$ contains any integer greater than $n(m)$.

Second observe that if $\{k(j)\}_{j=0}^{r}$ is a reasonable sequence with at most one $j \geqslant 1$ satisfying $n(i-1)<k(j) \leqslant n(i)$ for each $i \geqslant 1$, then increasing each $k(j)$ to the nearest $n(i)$ with $k(j) \leqslant n(i)$, produces another reasonable sequence. Doing this to each element of $T$ preserves all the properties above and we may assume that if the integer $k$ is an element of $T$ then for some $i, k=n(i)$.

Let $S$ be the sublist of $T$ of reasonable sequences $\{k(j)\}_{j=0}^{r}$ with $k(1)=n(1)$. From the above, $S$ has exactly $q$ elements. Suppose $\{k(j)\}_{j=0}^{r}$ is in $S$. Since this is a reasonable sequence, $r \leqslant k(1)=n(1)<m$. Thus for some $1 \leqslant i \leqslant m$, there is an $n(i) \notin\{k(j)\}_{j=0}^{r}$. There is some $\left\{k^{\prime}(j)\right\}_{j=0}^{s}$ in $T \backslash S$ with $n(i) \in\left\{k^{\prime}(j)\right\}_{j=0}^{s}$, since $q$ elements of $T$ have $n(i)$ as an element. If $r<n(1)$, we can add $n(i)$ to $\{k(j)\}_{j=0}^{r}$ and delete it from $\left\{k^{\prime}(j)\right\}_{j=0}^{s}$ producing two new reasonable sequences and preserving the above properties of $T$. Repeating as necessary, we may assume that if $\{k(j)\}_{j=0}^{r}$ is in $S$, then $r=n(1)$.

For each $1 \leqslant i \leqslant m$, let $s(i)$ be the number of reasonable sequences in $S$ that contain the integer $n(i)$. The function $s(i)$ has the following properties.

(1) $s(1)=q$,

(2) $1 \leqslant i \leqslant m, s(i) \geqslant 1$,

(3) $\sum_{i=1}^{m} s(i)=q n(1)$.

(Statement (2) follows since $q>p / 2$ and $T$ is a counterexample.) We would like to add

(4) $s(i)$ is nonincreasing.

To see that we may assume (4), suppose $1<\xi<\eta \leqslant m$ are integers, so that $S(\xi)<S(\eta)$. Then there is at least one $\{k(j)\}_{j=0}^{n(1)}$ in $S$ which contains $n(\eta)$ but not $\eta(\xi)$. Also there must be at least one $\left\{k^{\prime}(j)\right\}_{j=0}^{r}$ in $T \backslash S$ which contains $\eta(\xi)$ but not $n(\eta)$. Form new sequences, $\{l(j)\}_{j=0}^{n(1)}=(\{k(j): 0 \leqslant j \leqslant n(1)\} \cup\{\eta(\xi)\}) \backslash\{n(\eta)\}$, 
and $\left\{l^{\prime}(j)\right\}_{j=0}^{r}=\left(\left\{k^{\prime}(j): 0 \leqslant j \leqslant r\right\} \cup\{n(\eta)\}\right) \backslash\{n(\xi)\}$. The first is reasonable since $k(1)=n(1)<n(\xi)$ and the second is reasonable since $k^{\prime}(1) \leqslant n(\xi)<n(\eta)$. Replacing $\{k(j)\}_{j=0}^{n(1)}$ by $\{l(j)\}_{j=0}^{n(1)}$ and $\left\{k^{\prime}(j)\right\}_{j=0}^{r}$ by $\left\{l^{\prime}(j)\right\}_{j=0}^{r}$ preserves the properties of $T$ and $S$ above and the new $s(\xi)$ is one larger, while the new $s(\eta)$ is one smaller. Continuing, in some systematic way, eventually (4) will be true.

A useful fact is that for integers $1 \leqslant q \leqslant m$ and an integer-valued function $s(i)$ on $1 \leqslant i \leqslant m$ satisfying (1), (2), (3) and (4), then there is a $q$-element list $S$ of reasonable sequences of the form $\{k(j)\}_{j=0}^{n(1)}$ so that $s(i)$ is the number of reasonable sequences in $S$ that contain $n(i)$. This can be proved by induction on $q$. Indeed, let $\xi$ be the last integer $\leqslant m$ so that $s(\xi)=q$. Conditions (2) and (3) imply $\xi<n(1)$ since $n(1)<m$. Thus we define a reasonable sequence by

$$
k(j)= \begin{cases}n(j) & \text { if } 0 \leqslant j \leqslant \xi \\ n(m+j-n(1)) & \text { if } \xi<j \leqslant n(1) .\end{cases}
$$

If $s(i)$ is decreased by one at each $i$ where $n(i)$ is in the sequence $\{k(j)\}_{j=0}^{n(1)}$, the new function $s^{\prime}(i)$ satisfies (1)-(4) with $q^{\prime}=q-1$ and some $m^{\prime} \leqslant m$. The inductive hypothesis concludes the construction.

Define $\xi(i)$, for $1 \leqslant i \leqslant q$, to be the smallest integer $\leqslant m$, so that $s(\xi(i))=i$, if it exists, and $\xi(i)=m$ if it does not exist. (If $p$ is even, $s(m) \geqslant 2$ and $\xi(1)$ must be defined to be $m$.) If $T$ and $T^{\prime}$ are counterexamples satisfying the above with $\xi(i)$ and $\xi^{\prime}(i)$ as above we will say $T<T^{\prime}$ if $\xi(q)<\xi^{\prime}(q)$ or if $\xi(i)=\xi^{\prime}(i)$ for $q \geqslant i>j \geqslant 1$ and if $\xi(j)>\xi^{\prime}(j)$. Pick $T$ to be a maximal counterexample in this order.

There is a reasonable sequence $\left\{k^{\prime}(j)\right\}_{j=0}^{r}=R$ in $T \backslash S$ with $k^{\prime}(1)=n(\xi(q)+1)$. Consider the integer $n(\xi(j)), q>j \geqslant 1$, and suppose $n(\xi(j)) \notin R$. Then

$$
(R \cup\{n(\xi(j))\}) \backslash\{n(\xi(q)+1)\}=R^{\prime}
$$

is a reasonable sequence since $n(\xi(j))>n(\xi(q)+1)$. Let $s^{\prime}(i)$ be $s(i)$ except at $n(\xi(q)+1)$, where it is increased by one, and at $n(\xi(j))$, where it is decreased by one, note $s^{\prime}(i)$ satisfies (1)-(4). Let $T^{\prime}=S^{\prime} \cup\left((T \backslash(S \cup\{R\})) \cup\left\{R^{\prime}\right\}\right)$, where $S^{\prime}$ is a list of reasonable sequences with function $s^{\prime}(i)$. It follows that $T^{\prime}$ is a counterexample and $T^{\prime}>T$. Therefore, $n(\xi(j)) \in R$ for $q>j \geqslant 1$.

Now let $i$ be an integer with $\xi(j+1)<i \leqslant \xi(j)$ for some $j, q>j \geqslant 1$. The claim is that the integer $n(i) \in R$. Suppose $n(i) \notin R$. Since $s(i)=s(\xi(j))$ and $\xi(j) \in R$, there must be a reasonable sequence $\{k(j)\}_{j=0}^{r}$ in $T \backslash S$ that contains $n(i)$ but not $n(\xi(j))$. Since $n(i)<n(\xi(j))$ and $n(\xi(q)+1)<n(i)$, we can interchange these values between $R$ and $\{k(j)\}_{j=0}^{s}$ as we did in the proof of (4) obtaining a new counterexample. But for this counterexample $T$ the last paragraph shows that $T$ is not maximal. Therefore $n(i) \in R$.

It follows that $n(i) \in R$ for each $i$, so that $\xi(q)<i \leqslant \xi(1)=m$. Clearly $\xi(q) \leqslant$ $n(1)$. Thus if $R=\left\{k^{\prime}(j)\right\}_{j=0}^{r}$, then $r \leqslant n(1)+1$ and

$$
k^{\prime}(r) \leqslant n(n(1)+r)=n(2 n(1)+1)
$$

since $R$ is reasonable. Since $n(m) \in R, m \leqslant 2 n(1)+1<2 n(1)+2=m$. This contradiction completes the proof of the lemma and Theorem 4.3. 


\section{REFERENCES}

1. Z. Altshuler, Uniform convexity in Lorentz sequence spaces, Israel J. Math. 20 (1975), 260-274.

2. S. F. Bellenot, Uniformly complemented $l_{p}^{n}$ 's in quasi-reflexive Banach spaces, Israel J. Math. 39 (1981), 234-246.

3. A. Brunel and L. Sucheston, Equal signs additive sequences in Banach spaces, J. Funct. Anal. 21 (1976), 286-304.

4. P. G. Casazza and R. H. Lohman, A general construction of spaces of the type of R. C. James, Canad. J. Math. 27 (1975), 1263-1270.

5. W. J. Davis and J. Lindenstrauss, The $l_{1}^{n}$ problem and degrees of non-reflexivity. II, Studia Math. 58 (1976), 179-196.

6. R. C. James, Separable conjugate spaces, Pacific J. Math. 10 (1960), 563-571.

7. __ A non-reflexive Banach space that is uniformly nonoctahedral, Israel J. Math. 18 (1974), $145-155$

8. __ Banach spaces quasi-reflexive of order one, Studia Math. 60 (1977), 157-177.

9. __ Nonreflexive spaces of type 2, Israel J. Math. 30 (1978), 1-13.

10. Bor-Luh Lin and R. H. Lohman, On generalized James quasi-reflexive Banach spaces.

11. J. Lindenstrauss and C. Stegall, Examples of separable spaces which do not contain $l_{1}$ and whose duals are non-separable, Studia Math. 54 (1975), 81-105.

12. J. Lindenstrauss and L. Tzafriri, Classical Banach spaces. I. Sequence spaces, Ergebnisse der Math. und ihrer Grenzgebiete, vol. 92, Springer-Verlag, Berlin and New York, 1977.

13. J. C. B. Perrot, Thesis, Oxford University, 1976.

14. __ Transfinite duals of Banach spaces and ergodic super-properties equivalent to super-reflexivity, Quart. J. Math. Oxford Ser. (2) 30 (1979), 99-111.

Department of Mathematics and Computer Science, Clarkson College, Potsdam, New York 13676

Department of Mathematics and Computer Science, Florida State University, Tallahassee, FLORIDA 32306 (Current address) 